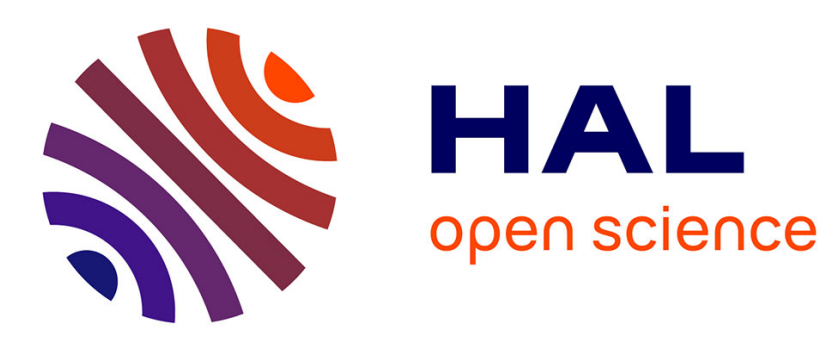

\title{
Suppression of underlying neuronal fluctuations mediates EEG slowing during general anaesthesia
}

Axel Hutt, Jeremie Lefebvre, Darren Hight, Jamie Sleigh

\section{To cite this version:}

Axel Hutt, Jeremie Lefebvre, Darren Hight, Jamie Sleigh. Suppression of underlying neuronal fluctuations mediates EEG slowing during general anaesthesia. NeuroImage, 2018. hal-02939283

\section{HAL Id: hal-02939283 \\ https://hal.inria.fr/hal-02939283}

Submitted on 18 Sep 2020

HAL is a multi-disciplinary open access archive for the deposit and dissemination of scientific research documents, whether they are published or not. The documents may come from teaching and research institutions in France or abroad, or from public or private research centers.
L'archive ouverte pluridisciplinaire HAL, est destinée au dépôt et à la diffusion de documents scientifiques de niveau recherche, publiés ou non, émanant des établissements d'enseignement et de recherche français ou étrangers, des laboratoires publics ou privés. 


\title{
1 Suppression of underlying neuronal fluctuations mediates EEG \\ 2 \\ slowing during general anaesthesia
}

3

4

9

\author{
Axel Hutt¹, Jérémie Lefebvre², Darren Hight ${ }^{3}{ }^{4}$, Jamie Sleigh ${ }^{3, *}$ \\ 1 Department FE 12 - Data Assimilation, Deutscher Wetterdienst, 63067 Offenbach am Main, Germany \\ Department of Mathematics and Statistics, University of Reading, Reading RG6 6AX,UK \\ ${ }^{2}$ Krembil Research Institute, University Health Network, Toronto, Ontario M5T 2S8, Canada \\ Department of Mathematics, University of Toronto, Toronto, Ontario M5T 2S8, Canada \\ Institute of Biomaterials and Biomedical Engineering, University of Toronto, Toronto, Ontario M5T \\ 2S8, Canada
}

${ }^{3}$ Department of Anaesthesiology, Waikato Clinical Campus, University of Auckland, Hamilton 3240, New Zealand

${ }^{4}$ Department of Anaesthesiology and Pain Therapy, University Hospital Bern, Inselspital, Bern, Switzerland

Short title: Random fluctuations slow anaesthesia EEG

Keywords : anaesthesia, noise, functional fragmentation, alpha-activity

\section{Corresponding author:}

Axel Hutt

Deutscher Wetterdienst

Department FE12- Data Assimilation

Frankfurter Strasse 135

63067 Offenbach am Main , Germany

Tel.: +496980622750 , Email: digitalesbad@gmail.com 


\section{Abstract}

31 The physiological mechanisms by which anaesthetic drugs modulate oscillatory brain activity

32 remain poorly understood. Combining human data, mathematical and computational analysis of

33 both spiking and mean-field models, we investigated the spectral dynamics of encephalographic

34 (EEG) beta-alpha oscillations, observed in human patients undergoing general anaesthesia. The

35 effect of anaesthetics can be modelled as a reduction of neural fluctuation intensity, and/or an

36 increase in inhibitory synaptic gain in the thalamo-cortical circuit. Unlike previous work, which

37 suggested the primary importance of gamma-amino-butryic-acid(GABA) augmentation in causing a

38 shift to low EEG frequencies, our analysis demonstrates that a non-linear transition, triggered by a

39 simple decrease in neural fluctuation intensity, is sufficient to explain the clinically-observed

40 appearance - and subsequent slowing - of the beta-alpha narrowband EEG peak. In our model,

41 increased synaptic inhibition alone, did not correlate with the clinically-observed encephalographic

42 spectral changes, but did cause the anaesthetic-induced decrease in neuronal firing rate. Taken

43 together, our results show that such a non-linear transition results in functional fragmentation of

44 cortical and thalamic populations; highly correlated intra-population dynamics triggered by

45 anaesthesia decouple and isolate neural populations. Our results are able to parsimoniously unify

46 and replicate the observed anaesthetic effects on both the EEG spectra and inter-regional

47 connectivity, and further highlight the importance of neural activity fluctuations in the genesis of

48 altered brain states. 
52 General anaesthesia is a widely used medical procedure in today's clinical practice. One of the main 53 obstacles in the optimization of general anaesthesia is the limited understanding of the 54 physiological mechanisms behind anaesthetic actions and their consequences on neural 55 information processing.

56 One of the prominent effects of most commonly used anaesthetic drugs is the induction of 57 characteristic changes in the spectral properties of electrophysiological activity across cortical 58 brain areas and spanning multiple frequency bands $(1,2,3,4)$. These spectral changes have been 59 used as the basis for many monitoring indices of electroencephalographic data (EEG) measured 60 empirically during anaesthesia (5), and as such constitute one of the most reliable biomarkers of 61 the brain's arousal state. One of the most salient spectral changes observed in clinical practice is the 62 generation of prominent oscillatory activity in the $\beta$-frequency band $(12 \mathrm{~Hz}-20 \mathrm{~Hz})$ and its 63 subsequent gradual decrease to the $\alpha$-frequency band ( $8 \mathrm{~Hz}-12 \mathrm{~Hz}$ ) with increasing anaesthetic 64 concentration (6).

65 This slowing down in oscillatory neural activity has also been shown to correlate strongly with 66 patients' behavioural state. Indeed, in parallel to the encephalographic deceleration from $\beta$ - to $\alpha$ 67 activity, subjects' behavioural state changes from a sedated state to one of loss of responsiveness 68 which we may equate with loss of connected consciousness (LOC).

69 Previous theoretical and computational studies have developed neural network models $(7,8)$ as 70 well as neural mass models $(9,10)$ to reproduce both $\beta$ - and $\alpha$-activity as observed across the 71 different states of anaesthesia. While insightful, these studies use network models with the limiting 72 assumptions of single anaesthetics acting on specific synaptic receptors or isolated ion channels, 73 and do so symmetrically across all neurons in a given circuit. From a practical perspective 
74 however, mixtures of different classes of anaesthetic agents - as opposed to only one agent - are

75 almost always used in clinical settings to induce general anaesthesia. These combinations of

76 anaesthetic drugs are known to interact with each other (11), and are thus likely to impact neurons

77 and neuronal populations in heterogeneous ways. In the absence of known physiological

78 mechanisms and detailed information about anaesthetics interactions, it remains highly challenging

79 to model, yet properly characterize, general anaesthesia from the single neuron perspective.

80 In contrast to previous studies where the characteristic slowing of EEG rhythms is caused by

81 changes in single neuron properties (often modelled as slowing of the inhibitory post-synaptic

82 potential decay time), we here propose an alternative mechanism: that the characteristic EEG

83 slowing is driven by system-level interactions. Using human data, and based on our mathematical

84 and computational analysis of both spiking and mean-field models, we have found that the

85 oscillatory transitions, commonly observed across sedation stages, appear to be a characteristic

86 signature of non-linear systems undergoing a phase transition: brain dynamics switches between

87 different dynamical states, accompanied by an amplification of coherent activity. To provide deeper

88 insight into the neural mechanism of anaesthetics from a circuit point of view, our work shows that

89 anaesthetic action results in an effective decrease in broadband neural activity i.e. the neural

90 activity exchanged between neural structures involved becomes more regular and coherent. This

91 hypothesis is in full agreement with converging lines of evidence showing that neural activity in

92 awake human subjects and animals is highly irregular compared to activity under anaesthesia;

93 which has very limited spatio-temporal information content $(3,12-15)$. A previous study (16)

94 demonstrated nicely that spatial frontal coherence in the $\alpha$-frequency increases markedly at the

95 point when subjects lose consciousness.

96 We considered a thalamo-cortical circuit of spiking neurons subjected to additive external random

97 fluctuations. When the fluctuation variance increases (as occurs during emergence from 
anaesthesia), mathematical and computational analysis reveals an oscillatory transition that is a characteristic signature of non-linear systems. With decreasing variance of fluctuations (as occurs with increasing depth of anaesthesia), the opposite occurs and the system exhibits an amplification of coherent dynamics. The corresponding connectivity analysis reveals enhanced intra-area synchrony but a simultaneous breakdown of inter-area synchrony. Taken together, our results support previously proposed hypotheses (17) in which anaesthesia represents a systemic isolation of neural populations via a switch from globally-driven to locally-driven activity. Taken together, our results show that circuit-scale interactions - as opposed to single neuron properties - are mandatory to replicate the slowing down of EEG activity observed in human clinical data.

\section{Materials and Methods}

\section{Experimental data}

Experimental EEG data have been obtained from two patients recorded for a previous observational study (6). This had institutional ethics approval (from the New Zealand Health and Disability Ethics Committee, Ref. 12/CEN/56, 2013) and written informed consent. These patients were both female (aged 29 and 77), and had anaesthesia initiated by a single dose of propofol, and subsequently maintained by the volatile anaesthetic sevoflurane during their surgery.

The data under study was recorded from a frontal position $\left(\mathrm{Fp}_{1 / 2}-\mathrm{Fp}_{\mathrm{z}}\right.$ in the $10-20$ system $)$ using a Bispectral Index depth of anaesthesia monitor (BIS ${ }^{\circledR}$, Covidien, Boulder, CO, USA) at a sampling frequency of $128 \mathrm{~Hz}$, which was then down-sampled to $100 \mathrm{~Hz}$. High and low pass non-phase shifting Butterworth filters were applied to the data (at 0.25 and $48.5 \mathrm{~Hz}$ cutoffs respectively, both set at $3 \mathrm{~dB}$ ). We have analyzed the temporal evolution of the frequency content of experimental data 
120 by computing its spectrogram with window width $\mathrm{T}=10$ s yielding a frequency resolution of $0.1 \mathrm{~Hz}$ 121 and window overlap of $0.1 \mathrm{~s}$.

\section{Anaesthetic action of the ascending arousal system}

124 Anaesthetics affect neural communication by modifying synaptic receptor and ion channel 125 properties. It has been shown previously (18) that increasing anaesthetic concentrations diminish 126 thalamic input to the cortex and reduces thalamic and cortical firing activity caused by reduced

127 activation in the ascending arousal system (AAS, 19). Since the input to the cortex and the thalamus 128 is rather complex and not known in all detail, we consider external input as random fluctuations 129 with a certain mean and variance. Our underlying model considers neural population activity and 130 the random fluctuations represent an uncorrelated synaptic bombardment with high input 131 population firing.

132 To estimate the anaesthetic action on the amplitude of those random fluctuations, we consider a 133 simple neural network describing anaesthetic action in the AAS $(19,20)$. Several clinically relevant 134 anaesthetics affect GABAergic synaptic and extra-synaptic receptors in the AAS inducing phasic and 135 tonic inhibition, respectively $(2,21)$. For instance, GABAergic synaptic receptors are present in the 136 AAS-structures brainstem, hypothalamus and the basal forebrain $(2,21)$ and anaesthetics prolong

137 their phasic response. Extra-synaptic receptors occur primarily in sub-cortical structures such the 138 brainstem, hypothalamus and thalamus $(10,22)$ and anaesthetics enhance the tonic inhibitory 139 current.

140 The AAS exhibits a rather complex network of interacting structures that inhibit each other

141 according to diverse direct and indirect actions. Previous experimental and theoretical studies of 142 phasic and tonic inhibition (23) have shown that increasing the synaptic time constant and 
143 decreasing the pre-synaptic firing rate decreases the synapses phasic response variance. This

144 indicates decreased response mean and variance with increased anaesthetic action by prolonged 145 post-synaptic phasic response and enhanced pre-synaptic firing reduction by tonic inhibition. To

146 show this anaesthetic impact, we consider anaesthetic actions on both phasic and tonic inhibition in 147 our simplified model of the AAS. To illustrate the phasic and tonic inhibition separately and clarify 148 their action, we distribute phasic and tonic receptors over different populations. A chain of three 149 neural populations of spiking neurons (Fig.1(a)) reflect the functional structure of AAS. The first 150 two populations exhibit tonic and phasic inhibition and the last population sums up the output 151 providing the random fluctuations (Fig.1(a)). These fluctuations may represent the input to 152 thalamic structures or to the cortex.

153 The first population (neurons $t$ in Fig.1(a)) includes uncoupled Leaky Integrate-and-Fire neurons 154 (LIF) of number $N=80$ with tonic inhibition $(23,24)$. The neurons are driven by a constant input 155 current. Such neurons may be located in brainstem structures, such as the locus coeruleus or 156 parabrachial nucleus that project to a large part of the cortex and thalamus. Their membrane 157 potential $V_{n}$ obeys

$$
C \frac{d V_{n}}{d t}=g_{l}\left(E_{l}-V_{n}\right)+g_{t}\left(E_{t}-V_{n}\right)+I, \quad n=1, \ldots, N
$$

158 for $V_{n}<\theta_{n}$. The thresholds $\theta_{n}$ are random and uniformly distributed about the mean threshold $159 \theta=-58 \mathrm{mV}$ in the range $[-59.5 \mathrm{mV} ;-56.5 \mathrm{mV}]$. The reset potential is $V_{\text {reset }}=-68 \mathrm{mV}$ and an $8 \mathrm{~ms}$ 160 refractory time is chosen. The other parameters are the capacity $C=33 n F$, the constant leak 161 conductance $g_{l}=22 n S$, the leak reversal potential $E_{l}=-76 \mathrm{mV}$ and the synaptic reversal potential $162 E_{t}=-76 \mathrm{mV}$. The external current is $I=8 n \mathrm{~A}$. The tonic conductance takes values $g_{t}=0 n S$ $163, g_{t}=200 n S$ and $g_{t}=400 n S$ that represent low, medium and high tonic anaesthetic action, 164 respectively. In the numerical simulations, initial conditions are random and distributed uniformly 
165 in the interval $\left[V_{\text {reset }}-15 \mathrm{mV} ; V_{\text {reset }}+15 \mathrm{mV}\right]$. Figure $1(\mathrm{~b})$ shows raster plots for the three 166 anaesthetic levels. The larger the inhibitory tonic current, the lower is the firing rate in accordance 167 to previous studies $(23,24)$.

168 These neurons emit spikes targeting inhibitory GABAergic synapses (neurons $i$ in Fig.1(a)) of 169 number $N$ with an exponential response function of time scale $\tau$. Such synapses may be located in 170 the hypothalamus, e.g. in the ventrolateral preoptic nucleus, receiving projections from the 171 brainstem. Hence the inhibitory response potential $I P S P_{m}$ at post-synaptic neuron $m$ obeys

$$
\tau \frac{d I P S P_{m}}{d t}=-I P S P_{m}+s_{m}, m=1, \ldots, N
$$

172 where $s_{m}=1 \mathrm{mV}$ when pre-synaptic spikes arrive and $s_{m}=0$ otherwise. Anaesthetics prolong the 173 synaptic time scale and we choose $\tau=200 \mathrm{~ms}, \tau=400 \mathrm{~ms}$, and $\tau=600 \mathrm{~ms}$ for low, medium and 174 high anaesthetic impact, respectively. Figure 1(c) presents the sum of these synaptic responses $175 \operatorname{IPSP}(t)=\sum_{m=1}^{N} I P S P_{m}(t)$ and reveals reduced magnitude and bias of post-synaptic currents with 176 increased anaesthetic action. 
(A)

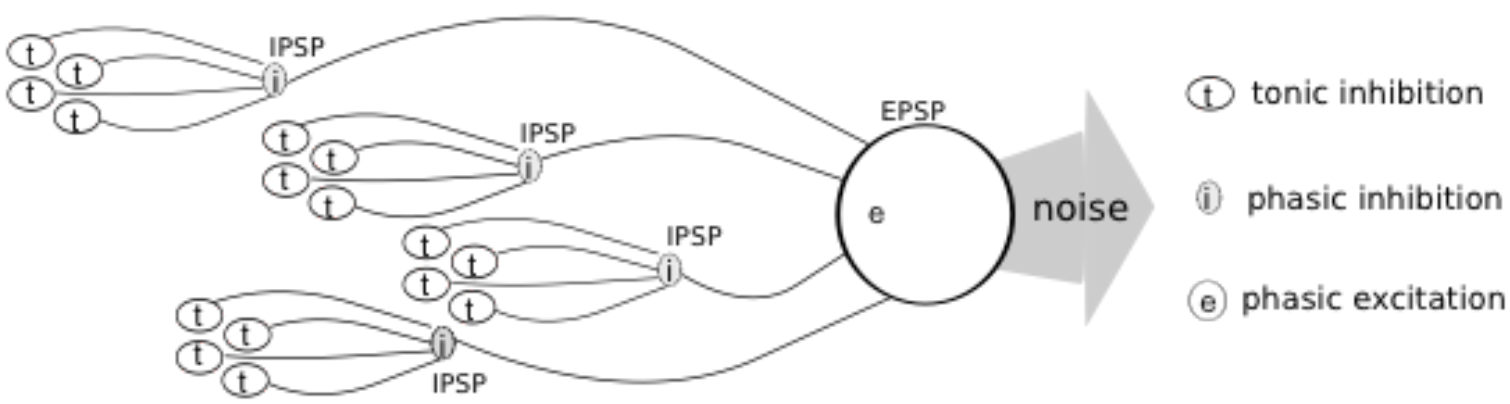

(B)

(D)
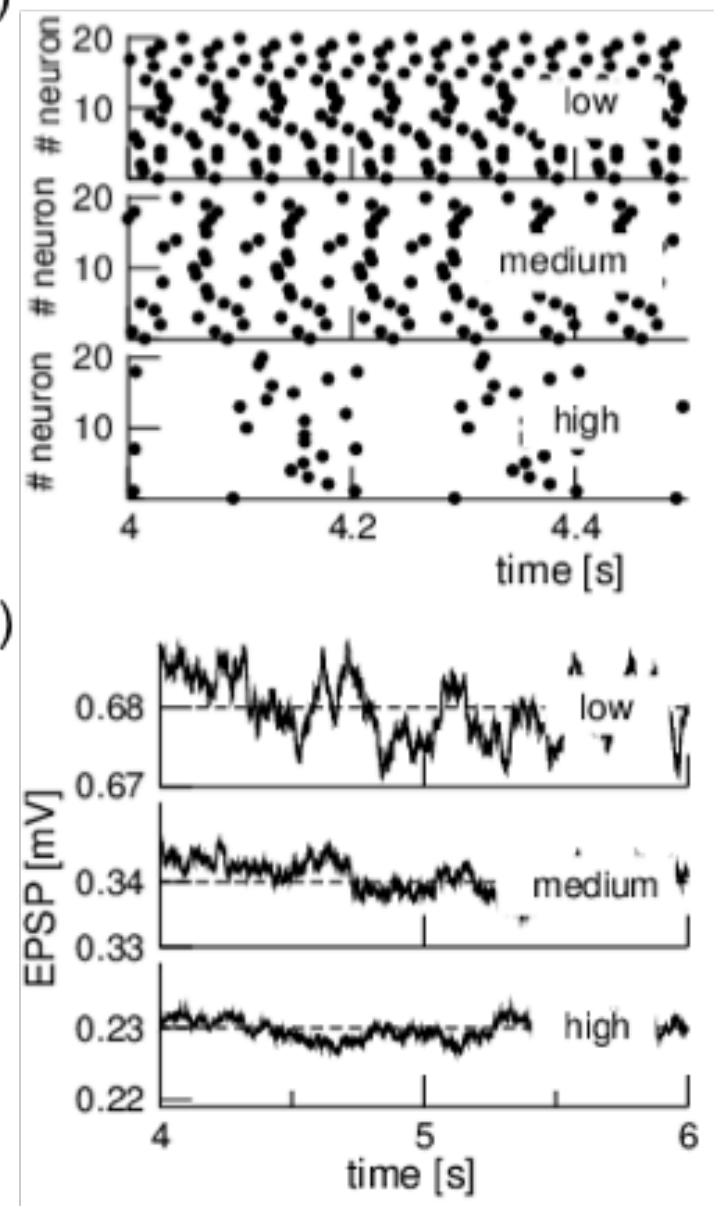

(C)

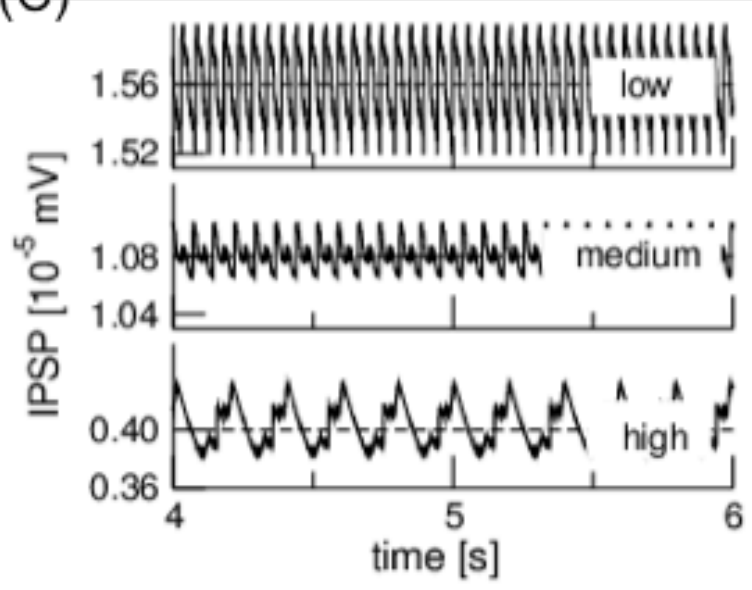

(E)

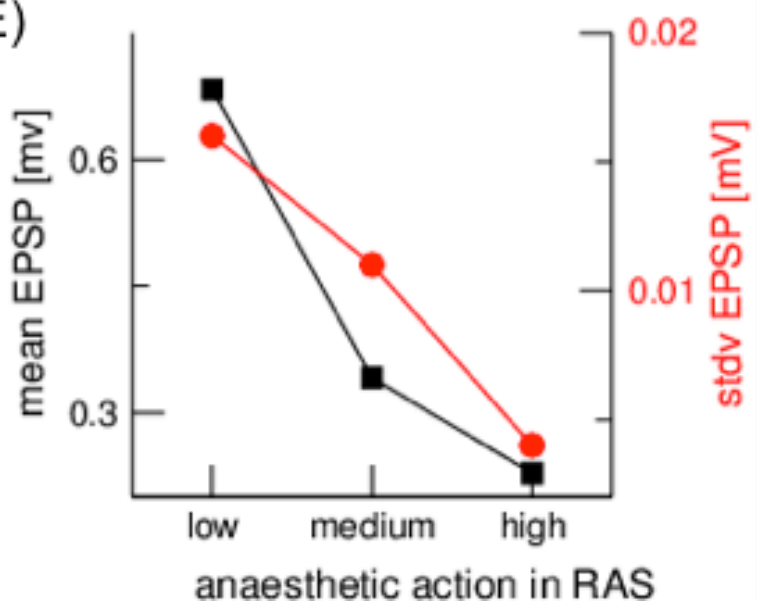

Figure 1: Effect of the ascending arousal system on cortical and thalamic activity. (A) Topology of the simulated network. (B) Spike trains of the output of tonic inhibition neurons targeting phasic inhibition neurons for different anaesthetic action levels (C) Inhibitory post-synaptic potentials

181 (IPSP) evoked by spike trains in (B). (D) Excitatory post-synaptic potentials (EPSP) that describes 
182 the noise applied to the cortico-thalamic network. (E) Both mean and standard deviation of EPSP

183 decrease with the anaesthetic action level.

184 The summed-up inhibitory current triggers the spike discharge in the post-synaptic neuron what is 185 modeled as a Poisson neuron with firing rate $\lambda(t)=f[\operatorname{IPSP}(t)]$ with the transfer function $186[U]=\frac{100 \mathrm{~Hz}}{1+\mathrm{e}^{-(U-\vartheta)}}, \vartheta=8 \mu V$. We consider $\mathrm{M}=40$ of such Poisson neurons whose spike discharges 187 target excitatory synapses (neuron $e$ in Fig.1(a)) with exponential response function and constant 188 time scale of $20 \mathrm{~ms}$. Such synapses may be located in the thalamus or the cortex. The sum of these 189 excitatory responses EPSP represents the random fluctuations. Figure 1(d) demonstrates that 190 stronger anaesthetic action decreases the magnitude of EPSPs and its bias. Since this EPSP 191 describes the input of the AAS to thalamus and cortex, we summarize that increasing anaesthetic 192 action reduces the variance of input fluctuations and its bias. These results are summarized in 193 Fig.1(e). This finding is consistent with recent experimental evidence in rats showing that 194 somatosensory and primary motor neurons in the anaesthetic-induced isoelectric state are fully 195 responsive to external stimuli (55). This finding is affirmed qualitatively for humans in the same 196 study. Since anaesthetic isoelectric states emerge under deep anaesthesia, this result demonstrates 197 that neurons are not strongly inhibited in deep anaesthesia as it would be the case if synaptic 198 inhibition alone would be present. We argue that anaesthetic action rather diminishes the input 199 from AAS to these neurons and, in this way, retain their responsiveness.

200 In the following we modify the fluctuation variance and keep the bias constant for the frontal 201 cortico-thalamic model. This approximation is reasonable since additional studies (not shown) 202 revealed that the major results in the present work are invariant for small anaesthetic-induced bias 203 adaptation. In the occipital cortico-thalamic model, we modify both the fluctuation variance and the 204 bias. We find that the additional bias reduction is mandatory to reproduce qualitatively the occipital 
power reduction. For simplicity, mentioning the fluctuation variance in the following discussion of the occipital model always implies a synchronous bias reduction.

\section{Anaesthetic action in the frontal cortico-thalamic network}

209 The relation proposed above between anaesthetic action and input variance does not specify in

210 detail the relation between fluctuation variance and anaesthetic action of different anaesthetic

211 agents in the cortex and sub-cortical structures. We hypothesize that this relationship is an effective

212 approximation of anaesthetic action and it is valid for various combinations of anaesthetic agents.

213 Our analysis suggests that this represents one of the major mechanisms of such pharmacological

214 agents on thalamocortical populations.

215 We assume that neuron ensembles in the cortex and thalamus are subjected to endogenous,

216 background sources of inputs $A_{k}^{j}(t)=\sqrt{2 D} \xi_{k}^{j}(t)$ with $k=e, I$, th, rth for the cortical excitatory (e),

217 cortical inhibitory (i), thalamic lateral geniculate nucleus (th) and thalamic reticular (rtn)

218 population (cf. Fig.2(A)). Here, $\xi_{k}^{j}(t)$ are independent random Gaussian processes with identical

219 variance. Anaesthetic action in the cortex and thalamus is modeled by changing the level of noise or

220 fluctuation variance D in the system with increasing anaesthetics concentration in accordance to

221 the AAS-model. In numerical experiments, the variance either obeys the piece-wise exponential

222 laws

$$
D(t)=D_{1} e^{-t / \tau_{n}}+D_{0}, 0 \leq t_{1}
$$$$
D(t)=D_{2}, t_{1}<t \leq t_{2},
$$ 


$$
D(t)=-m t+D_{5}, 0 \leq t \leq t_{1}
$$

228 with $D_{0}>0, m>0$. For Eqs. (1), we choose parameters such that $(0)=1.01, D_{2}=0.01, D\left(t_{3}\right)=$

2290.04 with $t_{1}=t_{3} / 2, t_{2}=0.6 t_{3}, t_{3}=32.768 \mathrm{~s}, \tau_{n}=(32786 / 0.16) \mathrm{s}$. In the linear model in Eq. (2)

$230 D_{5}=0.345, \mathrm{~m}=0.005$ with $t_{1}=32.768 \mathrm{~s}$. Here, the temporal duration in both simulations is $23132.768 s$.
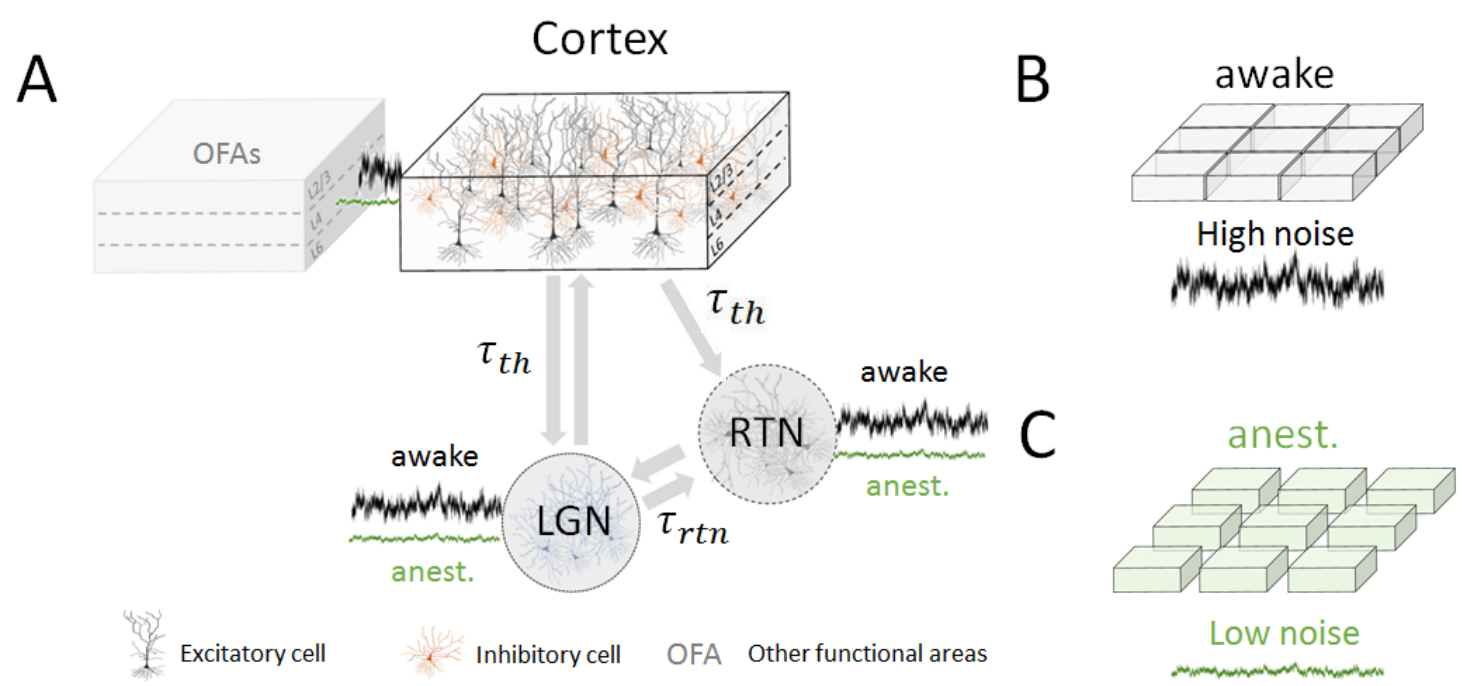

Low noise

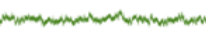

Figure 2: Illustration of thalamo-cortical network subjected to additive random fluctuations. (A) network topology (B) decreasing fluctuation variance functionally decouples the network.

We assume that this effective reduction of fluctuation variance represents the major effect of 237 anaesthetics on the cortico-thalamic circuit. In addition to the effect of fluctuations, it has been 238 shown in numerous experimental studies that a large variety of anaesthetic drugs inhibits neural 
239 activity by action on GABAergic synaptic receptors. To take into account this synaptic effect, we

240 included a prolongation of the synaptic decay time scale of inhibitory GABAergic receptors while

241 retaining the amplitude of the synaptic inhibitory impulse response. Mathematically the synaptic

242 decay time scale of GABAergic synapses obey

$$
\tau_{i}(t)=\tau_{i}^{0} p(t)
$$

244 When utilizing the exponential fluctuation variance model, the factor synaptic decay time scale 245 factor obeys the linear law

$$
\begin{aligned}
& p(t)=1+2 t / t_{1}, 0 \leq t_{1}, \\
& p(t)=3, t_{1}<t \leq t_{2}, \\
& p(t)=3-2\left(t-t_{2}\right) /\left(t_{3}-t_{2}\right), t_{2}<t \leq t_{3}
\end{aligned}
$$

249 with the same times $t_{1}, t_{2}$ and $t_{3}$ as in (1). The corresponding the linear model is $p(t)=1+$

$2502 t / t_{1}, 0 \leq t_{1}$. This anaesthetic model is motivated by a previous study based on experimental data 251 (51).

\section{Anaesthetic action in the occipital cortico-thalamic network}

253 The occipital anaesthetic action model is very similar to the frontal model. Since not much is known

254 about the projection targets of the AAS to the occipital cortex, we consider a diffusive projection to 255 the cortex via the basal forebrain $(19,56)$ but neglect AAS stimulation of thalamic structures. To 256 illustrate the spectral power dynamics with decreasing AAS activity, we assume the fluctuation 257 variance $D$ to decrease in time according to

$$
D(t)=D_{1} e^{-t / \tau_{n}}+D_{0}, 0 \leq t \leq t_{1}
$$


with $t_{1}=32.768 \mathrm{~s}, D(0)=0.06, \tau_{n}=(32786 / 0.16) \mathrm{s}$ and $D_{0}=0.0006$. The excitatory

259 endogenous, fluctuating background input is $A_{e}^{j}(t)=\sqrt{2 D} \xi_{k}^{j}(t)$, whereas other time-dependent

260 input $A_{i}^{j}(t)=A_{t h}^{j}(t)=A_{r t n}^{j}(t)=0$. This is different to the frontal model, where all cortical and 261 thalamic structures are driven by the AAS. Moreover, the mean excitatory input from AAS obeys the 262 Hill equation

$$
I_{e}(t)=-0.5-3 /\left(1+\left(0.7 t_{1} / t\right)^{4}\right), 0 \leq t \leq t_{1}
$$

264 that is a decreasing sigmoidal function with turning point at $t=0.7 t_{1}$. The mean input to the other 265 structures $I_{i}, I_{t h}, I_{r t n}$ remain constant. To consider anaesthetic effects on synaptic inhibition, the 266 synaptic time scale factor increases over time according to

$$
p(t)=1+2 /\left(1+\left(1.5 t_{1} / t\right)^{4}\right), 0 \leq t \leq t_{1}
$$

\section{Spiking frontal model}

269 To understand the underlying neural mechanism of changes in EEG spectral features, we 270 investigated a thalamo-cortical hybrid network model of Amari-type (25). The network is built of

271 recurrently connected neural ensembles of excitatory pyramidal neurons (e) and inhibitory 272 interneurons (i) interacting with thalamic relay (th) neurons in the Lateral Geniculate Nucleus 273 (LGN) and thalamic reticular neurons (rtn), cf. Fig.2 (A) .

274 Spiking activity of those neural ensembles follow the non-homogeneous Poisson processes

$$
\begin{gathered}
X_{e}^{j}(t) \rightarrow \text { Poisson }\left(f\left[u_{e}^{j}(t)\right]\right), X_{i}^{j}(t) \rightarrow \text { Poisson }\left(f\left[u_{i}^{j}(t)\right]\right) \\
X_{t h}^{j}(t) \rightarrow \text { Poisson }\left(f\left[u_{t h}^{j}(t)\right]\right), X_{r t n}^{j}(t) \rightarrow \text { Poisson }\left(f\left[u_{r t n}^{j}(t)\right]\right)
\end{gathered}
$$


277 where $X_{e, i, t h, r t n}^{j}(t)=\sum_{\left\{t_{l}\right\}} \delta_{e, i, t h, r t n}^{j}\left(t-t_{l}\right)$ is the spike train of the $j^{t h}$ ensemble patch in the 278 respective population. The ensemble response function $f[u]=(1+\exp \{-\beta(u-h)\})^{-1}$ represents 279 a saturating firing rate function with gain $\beta=150$ and threshold $h=0.1$. The average potentials of 280 ensemble $\mathrm{j}$ of excitatory and inhibitory neurons $u_{e}^{j}(t)$ and $u_{i}^{j}(t)$, respectively, and the thalamic 281 potentials $u_{t h}^{j}(t)$ and $u_{r t n}^{j}(t)$ obey

$$
\begin{gathered}
\alpha_{e}^{-1} \frac{d u_{e}^{j}(t)}{d t}=-u_{e}^{j}(t)+b v_{e}^{j}(t)+S_{e \rightarrow e}^{j}(t)+S_{i \rightarrow e}^{j}(t)+S_{t h \rightarrow e}\left(t-\tau_{t h}\right)+I_{e}+A_{e}^{j}(t) \\
\alpha_{i}^{-1} \frac{d u_{i}^{j}(t)}{d t}=-u_{i}^{j}(t)+b v_{i}^{j}(t)+S_{e \rightarrow i}^{j}(t)+S_{i \rightarrow i}^{j}(t)+S_{t h \rightarrow i}\left(t-\tau_{t h}\right)+I_{i}+A_{i}^{j}(t) \\
\alpha_{t h}^{-1} \frac{d u_{t h}^{j}(t)}{d t}=-u_{t h}^{j}(t)+b v_{t h}^{j}(t)+S_{e \rightarrow t h}^{j}\left(t-\tau_{t h}\right)+S_{r t n \rightarrow t h}^{j}\left(t-\tau_{r t n}\right)+I_{t h}+A_{t h}^{j}(t) \\
\alpha_{r t n}^{-1} \frac{d u_{r t n}^{j}(t)}{d t}=-u_{r t n}^{j}(t)+b v_{r t n}^{j}(t)+S_{e \rightarrow r t n}^{j}\left(t-\tau_{t h}\right)+S_{t h \rightarrow r t n}^{j}\left(t-\tau_{r t n}\right)+I_{r t n}+A_{r t n}^{j}(t) \\
a^{-1} \frac{d v_{e}^{j}(t)}{d t}=-v_{e}^{j}(t)+u_{e}^{j}(t) ; a^{-1} \frac{d v_{i}^{j}(t)}{d t}=-v_{i}^{j}(t)+u_{i}^{j}(t) \\
a^{-1} \frac{d v_{t h}^{j}(t)}{d t}=-v_{t h}^{j}(t)+u_{t h}^{j}(t) ; a^{-1} \frac{d v_{r t n}^{j}(t)}{d t}=-v_{r t n}^{j}(t)+u_{r t n}^{j}(t)
\end{gathered}
$$$$
285 \text { where } v_{n}^{j}(t) \text { refers to corresponding adaptation currents, } b \text { is the adaptation gain and } S_{n \rightarrow m}^{j} \text { are }
$$$$
286 \text { synaptic inputs from population } n \text { to population } m \text {. The variables } u_{n}^{j}(t) \text { represent the Local Field }
$$$$
287 \text { Potential that would be measured with an implanted electrode in population } n=e, i, t h, r t n \text {. The }
$$$$
288 \text { terms } I_{n} \text { are constant bias currents and } A_{n}^{j} \text { are random external input currents. Cortico-thalamic }
$$$$
289 \text { interactions are delayed by a propagation delay } \tau_{t h} \text {, while we have also included an interaction }
$$$$
290 \text { delay } \tau_{r t n} \text { between LGN and the reticular population. In addition, local, distance-dependent }
$$$$
291 \text { propagation delays are also included within the cortical populations. Using these, the resulting }
$$$$
292 \text { integrated synaptic inputs to the neurons are given by }
$$ 


$$
S_{n \rightarrow m}^{j}(t)=N_{n}^{-1} \sum_{k=1}^{N_{n}} W_{n \rightarrow m}^{j k} \cdot E_{n m}^{k}\left(t-\tau^{j k}\right) .
$$

293 The individual propagation delays are given by $\tau^{j k}=\Delta^{j k} / c$, where $c$ is a finite conduction velocity 294 and $\Delta^{j k}$ is the spatial distance between ensemble patch of number $\mathrm{j}$ and $\mathrm{k}$. Ensembles are 295 distributed randomly and uniformly within a one dimensional spatial domain $\Omega$ of length $|\Omega|$. 296 Average post-synaptic potentials $E_{n}^{k}(t)$ are computed as input spike trains convolved with 297 exponential synapses with time constant $\tau_{m}$

$$
E_{n m}^{k}(t)=\int_{o}^{t} X_{n}^{k}(s) \frac{1}{\tau_{m}} e^{-(t-s) / \tau_{m}} d s
$$

298 As pointed out above, $\tau_{i}=$ const or $\tau_{i}=\tau_{i}(t)$ in two studies. Recurrent synaptic weights amongst $299\left(W_{n \rightarrow n}^{j k}\right)$ and between $\left(W_{n \rightarrow m}^{j k}\right)$ excitatory and inhibitory populations exhibit sparse Gaussian profiles

$$
W_{n \rightarrow m}^{j k}(\rho)=\frac{w_{n \rightarrow m}^{o}(\rho)}{\sqrt{2 \pi \sigma_{n \rightarrow m}^{2}}} \exp \left[-\frac{\left(\Delta^{j k}\right)^{2}}{2 \sigma_{n \rightarrow m}^{2}}\right]
$$
where $w_{n \rightarrow m}^{o}(\rho)=w_{n \rightarrow m}^{o} \neq 0$ with probability $\rho$. Model constants are given in Table 1 . The numerical integration step is $\Delta t=0.001 \mathrm{~s}$ and all variables have been initialized in $t \in[-\tau ; 0]$ to $u_{n}, v_{m}=0$ with the maximum delay time $\tau$.

Table 1: Table of Model Parameters

\begin{tabular}{|c|c|c|c|c|c|r|}
\hline Symbol & Definition & Symbol & Definition & \\
\hline$\Omega$ & $\begin{array}{c}\text { Network spatial extent } \\
{[\mathrm{mm}]}\end{array}$ & 10 & $\rho$ & Connection probability & 0.2 \\
\hline$N_{e}$ & Number of excitatory cells & 800 & $w_{e \rightarrow e}^{o}$ & Synaptic connection strength & 12 \\
\hline$N_{i}$ & Number of inhibitory cells & 200 & $w_{e \rightarrow i}^{o}$ & Synaptic connection strength & -25 \\
\hline$N_{t h}$ & Number of thalamic cells & 200 & $w_{i \rightarrow e}^{o}$ & Synaptic connection strength & 25 \\
\hline$N_{r t n}$ & Number of reticular cells & 200 & $w_{i \rightarrow i}^{o}$ & Synaptic connection strength & -12 \\
\hline$\beta$ & $\begin{array}{c}\text { Neural response function } \\
\text { gain [1/mV] }\end{array}$ & 150 & $w_{e \rightarrow t h}^{o}$ & Synaptic connection strength & 5 \\
\hline
\end{tabular}




\begin{tabular}{|c|c|c|c|c|c|}
\hline$h$ & Firing rate threshold [Hz] & 1 & $w_{e \rightarrow r t n}^{O}$ & Synaptic connection strength & 5 \\
\hline$\tau_{m}$ & mean time constant $[\mathrm{s}]$ & 1 & $w_{t h \rightarrow e}^{o}$ & Synaptic connection strength & 35 \\
\hline$\alpha_{e}$ & $\begin{array}{c}\text { mean rate constant - } \\
\text { excitatory cortical cells } \\
{[\mathrm{Hz}]}\end{array}$ & 1.0 & $w_{t h \rightarrow i}^{o}$ & Synaptic connection strength & -10 \\
\hline$\alpha_{i}$ & $\begin{array}{c}\text { mean rate constant - } \\
\text { inhibitory cortical cells } \\
{[\mathrm{Hz}]}\end{array}$ & 1.5 & $w_{t h \rightarrow r t n}^{o}$ & Synaptic connection strength & 12 \\
\hline$\alpha_{t h}$ & $\begin{array}{l}\text { mean rate constant - } \\
\text { thalamic neurons }[\mathrm{Hz}]\end{array}$ & 0.2 & $w_{r t n \rightarrow t h}^{o}$ & Synaptic connection strength & 12 \\
\hline$\alpha_{r t n}$ & $\begin{array}{l}\text { mean rate constant - } \\
\text { reticular cells }[\mathrm{Hz}]\end{array}$ & 0.2 & $\overline{\sigma_{e \rightarrow e}^{2}}$ & $\begin{array}{l}\text { Synaptic connection range } \\
{[\mathrm{mm}]}\end{array}$ & 0.1 \\
\hline$a$ & $\begin{array}{l}\text { Neural adaptation rate } \\
\text { constant }[\mathrm{Hz}]\end{array}$ & 0.005 & $\sigma_{e \rightarrow i}^{2}$ & $\begin{array}{l}\text { Synaptic connection range } \\
{[\mathrm{mm}]}\end{array}$ & 0.1 \\
\hline$b$ & $\begin{array}{c}\text { Neural adaptation gain } \\
{[1 / \mathrm{s}]}\end{array}$ & 0.1 & $\sigma_{i \rightarrow e}^{2}$ & $\begin{array}{l}\text { Synaptic connection range } \\
{[\mathrm{mm}]}\end{array}$ & 2.5 \\
\hline$I_{e}$ & $\begin{array}{c}\text { Constant current bias } \\
{[\mathrm{mV} / \mathrm{s}]}\end{array}$ & 0 & $\sigma_{i \rightarrow i}^{2}$ & $\begin{array}{c}\text { Synaptic connection range } \\
{[\mathrm{mm}]}\end{array}$ & 2.5 \\
\hline$I_{i}$ & $\begin{array}{c}\text { Constant current bias } \\
{[\mathrm{mV} / \mathrm{s}]}\end{array}$ & 0 & $\sigma_{e \rightarrow t h}^{2}$ & $\begin{array}{l}\text { Synaptic connection range } \\
{[\mathrm{mm}]}\end{array}$ & 0.1 \\
\hline$I_{t h}$ & $\begin{array}{c}\text { Constant current bias } \\
{[\mathrm{mV} / \mathrm{s}]}\end{array}$ & 0.1 & $\sigma_{e \rightarrow r t n}^{2}$ & $\begin{array}{c}\text { Synaptic connection range } \\
{[\mathrm{mm}]}\end{array}$ & 0.1 \\
\hline$I_{r t n}$ & $\begin{array}{c}\text { Constant current bias } \\
{[\mathrm{mV} / \mathrm{s}]}\end{array}$ & -0.5 & $\sigma_{t h \rightarrow e}^{2}$ & $\begin{array}{c}\text { Synaptic connection range } \\
{[\mathrm{mm}]}\end{array}$ & 2.5 \\
\hline$\tau_{t h}$ & $\begin{array}{l}\text { Thalamo-cortical delay } \\
{[\mathrm{ms}]}\end{array}$ & 2 & $\sigma_{t h \rightarrow i}^{2}$ & $\begin{array}{l}\text { Synaptic connection range } \\
{[\mathrm{mm}]}\end{array}$ & 2.5 \\
\hline$\tau_{r t n}$ & $\begin{array}{c}\text { Reticular-thalamic delay } \\
{[\mathrm{ms}]}\end{array}$ & 2 & $\sigma_{t h \rightarrow r t n}^{2}$ & $\begin{array}{c}\text { Synaptic connection range } \\
{[\mathrm{mm}]}\end{array}$ & 2.5 \\
\hline$c$ & Conduction velocity [m/s] & 0.35 & $\sigma_{r t n \rightarrow t h}^{2}$ & $\begin{array}{l}\text { Synaptic connection range } \\
{[\mathrm{mm}]}\end{array}$ & 2.5 \\
\hline & & & $\mathrm{dt}$ & Integration time step [ms] & 1 \\
\hline
\end{tabular}

The network topology of the occipital model is identical to that of the frontal model. However, by virtue of the differences in intra-area neural structures and their different functional role in the brain, some parameters are different. The time scale of the intra-cortical excitatory and inhibitory adaption currents are chosen differently to

$$
a_{e}^{-1} \frac{d v_{e}^{j}(t)}{d t}=-v_{e}^{j}(t)+u_{e}^{j}(t) ; a_{i}^{-1} \frac{d v_{i}^{j}(t)}{d t}=-v_{i}^{j}(t)+u_{i}^{j}(t)
$$

311 with $a_{e}=1.0, a_{i}=0.1$. This specific parameter choice in the full parameter set fixes the oscillation 312 frequency at large fluctuation variance to about $10 \mathrm{~Hz}$. 


\section{Effective mean-field model}

315 To understand the macroscopic dynamics of the spiking neural network, it is insightful to consider

316 its spatially averaged dynamics. Statistical features and average population dynamics of the cortical

317 populations were exposed by investigating the mean-field formulation of the more fined grained 318 spiking model above, leaving aside the LGN and reticular neurons. Using a mean-field 319 approximation $(26,27)$, the mean dynamics of the excitatory and inhibitory cortical potentials $U_{e}$ 320 and $U_{i}$ can be shown to obey

$$
\begin{aligned}
& \alpha_{e}{ }^{-1} \frac{d U_{e}}{d t}=-U_{e}+b V_{e}+\bar{W}_{e \rightarrow e} F_{e}\left[U_{e}(t-\tau)\right]+\bar{W}_{i \rightarrow e} F_{i}\left[U_{i}(t-\tau)\right]+I_{e} \\
& \alpha_{i}{ }^{-1} \frac{d U_{i}}{d t}=-U_{i}+b V_{i}+\bar{W}_{e \rightarrow i} F_{e}\left[U_{e}(t-\tau)\right]+\bar{W}_{i \rightarrow i} F_{i}\left[U_{i}(t-\tau)\right]+I_{i}
\end{aligned}
$$

$$
a_{e}^{-1} \frac{d V_{e}}{d t}=-V_{e}+U_{e}
$$

where the fluctuation-corrected neural response function reads

$$
a_{i}^{-1} \frac{d V_{i}}{d t}=-V_{i}+U_{i}
$$

$$
\begin{gathered}
F_{k}[U]=\frac{1}{2}\left[1+\operatorname{erf}\left(\frac{U-h}{\sigma_{k} \sqrt{2}}\right)\right], \\
\sigma_{k}=-\sqrt{2} D\left(\frac{A^{2}}{2 \lambda_{+}}+\frac{2 A B}{\lambda_{+}+\lambda_{-}}+\frac{B}{2 \lambda_{-}}\right) \\
\lambda_{ \pm}=\left(-a_{k}-\alpha_{k} \pm \sqrt{\left(a_{k}-\alpha_{k}\right)^{2}+4 b a_{k} \alpha_{k}}\right) / 2, A=\sqrt{2 \pi} \frac{a+\lambda_{+}}{\lambda_{+}-\lambda_{-}}, B=-\sqrt{2 \pi} \frac{a+\lambda_{-}}{\lambda_{+}-\lambda_{-}} .
\end{gathered}
$$

329 Here, $\sigma_{k}$ is the standard deviation of colored random fluctuations about the mean-field neural 330 activity and each neuron behaves like a McCullogh-Pitts neuron with $\beta \rightarrow \infty$. Since $\sigma_{k} \sim D$, 331 decreasing the fluctuation variance renders the response function $F_{k}$ steeper. Previous studies 
332 have shown that this may diminish the frequency of synchronous oscillation (26). The coefficients

$333 \bar{W}_{n \rightarrow m}$ stand for the mean synaptic connectivity weights, computed by averaging all entries within 334 the associated random matrices.

335 To understand the solution dynamics of the mean-field model, we have calculated numerically the 336 stationary state of the mean-field model $\left(\bar{U}_{e}, \bar{U}_{i}, \bar{V}_{e}, \bar{V}_{i}\right)$ and studied numerically the temporal 337 dynamics of deviations $\left(u_{e}=U_{e}-\bar{U}_{e}, u_{i}=U_{i}-\bar{U}_{i}, v_{e}=V_{e}-\bar{V}_{e}, v_{i}=V_{i}-\bar{V}_{i}\right)$ from these states. 338 Parameters used are $\bar{W}_{e \rightarrow e}=2.21, \bar{W}_{e \rightarrow i}=4.58, \bar{W}_{i \rightarrow e}=-3.46, \bar{W}_{i \rightarrow i}=-1.69, b=0.1, a_{e}=a_{i}=5.0$ $339, \alpha_{e}=50.0, \alpha_{i}=100.0, I_{e}=0.1, I_{i}=0, h=0.1$.

\section{Spectral Analysis}

342 In the neural model, neuro-electric activity corresponds to a spatial summation of localized electric 343 potentials emanating from superficial cortical neurons. We modeled the electroencephalographic $344 E E G(t)$ signal as the weighted spatial average

$$
E E G(t)=N_{e}^{-1} \sum_{k=1}^{N_{e}} \phi_{e}^{k} u_{e}^{k}(t)+N_{i}^{-1} \sum_{k=1}^{N_{i}} \phi_{i}^{k} u_{i}^{k}(t)
$$

$\phi_{e, i}^{k}=[0,1]$ denote random, positive coefficients accounting for dispersion effects and other source

347 of observational noise. Spectral analysis in the time-frequency domain was performed applying 348 Morlet wavelet analysis with equidistant frequencies.

\section{Spike rate and spike coherence calculation}

351 The instantaneous firing rate is the estimated number of spikes of each neuron occurring in a time

352 window of $5 \mathrm{~ms}$. In our evaluation the spike rate depends on the linearly decreasing fluctuation 
353 variance and we applied a sliding window of width $\Delta D=0.01$. To evaluate statistically the change 354 of spike rate, we applied a non-parametric Kruskal-Wallis test with respect to the firing rate at 355 maximum fluctuation variance.

356 Mean spike coherence in the cortex was computed based on standard calculations (28), consisting 357 of two randomly selected neurons $i, j$ within the cortical population, and binning their respective 358 responses over a given time window $\Delta T$. Thus, for neuron $j, X_{j}^{b i n}(k \Delta T)=1$ if a spike occurred 359 during the interval $[k \Delta T,(k+1) \Delta T]$, and zero otherwise, where $k=T / \Delta \mathrm{T}$. Intra-area coherence $360 \theta(\Delta T)$ was then computed using

$$
\theta(\Delta T)=\frac{\sum_{T} X_{i}^{b i n} Y_{j}^{b i n}}{\sum_{T} X_{i}^{b i n 2} \sum_{T} Y_{j}^{b i n 2}}
$$

361 for two spike trains $X, Y$ in the same area. The spike activity under study is assumed to evolve on a 362 large time scale compared to the rapid spike dynamics. Consequently it is reasonable to average $363 \theta$ over a time window of duration $T$. In order to evaluate the evolution of the spike coherence with 364 changing fluctuation variance relative to the maximum fluctuation variance, we consider the 365 relative spike coherence $\theta / \theta_{0}$ where $\theta_{0}$ is the spike coherence with maximum variance $\mathrm{D}$. In the 366 simulations, we choose 100 randomly chosen pairs of neurons in the cortical E-population with a 367 small time window $\Delta T=5 \mathrm{~ms}$ and $T=1 \mathrm{~s}(\Delta D=0,00005$ and a range of fluctuation variances of $368 D=0.01$ in Figs. S3 and S4). Smaller time intervals $\Delta T$ retain the given results qualitatively. The 369 significance test is non-parametric of Kruskal-Wallis type and verifies the significance of the spike 370 coherence at various fluctuation variances compared to the maximum fluctuation variance.

371 In addition to the intra-area spike coherence, we compute the cross spike coherence between two 372 different areas where $X, Y$ are spike trains from the areas. The corresponding parameters are 373 identical to the case of intra-area spike coherence, but for 300 randomly chosen pairs of neurons 374 from the different populations. All given spike coherence estimates are ratios to the spike 
375 coherence at the largest fluctuation variance. The Kruskal-Wallis test verifies the significance of

376 cross coherences compared to cross coherences at the maximum fluctuation variance.

\section{$377 \quad$ Spike Field Coherence}

378 Spike Field Coherence (SFC) reflects the synchronization between spikes and corresponding 379 electric potentials (29). To estimate the SFC in the cortex at large and small fluctuations, we 380 compute it in both cases in a time window of $5 \mathrm{~s}$ in the $\delta-(0.5 \mathrm{~Hz}-4 \mathrm{~Hz}), \theta-(4 \mathrm{~Hz}-8 \mathrm{~Hz}), \alpha-(8 \mathrm{~Hz}-$ $38112 \mathrm{~Hz})$ and $\beta-(12 \mathrm{~Hz}-20 \mathrm{~Hz})$ frequency band. This standard measure estimates the coherence 382 between spikes and their corresponding electric potentials at the same cell averaged over all cells.

383 In addition, we estimate the spike-field coherence between cortical EEG and spiking activity in the 384 LGN cells and the reticular cell. The corresponding cross-SFC represents the coherence between 385 thalamic spikes and cortical EEG at the same time instant averaged over all cells.

\section{Global phase synchronization}

388 Phase synchronization (PS) is a measure of the phase consistency between two time series. In our 389 case, the PS between two populations in the network model is obtained by comparing the phase 390 between their respective mean potential activities. Typically PS is non-stationary and subject to 391 random fluctuations. The instantaneous phase-locking value (PLV) represents a statistical estimate 392 of the degree of constant phase relation between several time series in a certain time interval and a 393 certain frequency band (30). In fact, the PLV is the instantaneous circular variance of the phase 394 difference of two time series. We take up this definition and compute a global PS index (GPS) by $395 \quad(31)$

$$
G P S=\left|\frac{1}{N} \sum_{k=1}^{N} e^{-i \Delta \varphi_{k}}\right|^{2} \quad
$$


$\Delta \varphi_{k}$ is the phase difference in the time series pair $k$ at a certain time instance and $N$ is the number

398 of phase pairs considered. In practice, typically one averages over a set of pairs and time instances

399 in a certain time window. The Results section shows values of GPS gained by a Morlet wavelet 400 transform in the frequency range $[1 \mathrm{~Hz} ; 20 \mathrm{~Hz}]$ based on E-population Local Field Potentials down401 sampled to a sampling frequency of $50 \mathrm{~Hz}$. We have chosen randomly 20 pairs of neurons out of 402100 fixed randomly chosen neurons and averaged over 10 independent trials.

403 Instantaneous phases are defined mathematically in the case of non-vanishing instantaneous 404 amplitudes only and may be spurious for very low amplitudes. We define numerical phase values $405 \varphi_{l}$ from single time series $l$ in the E-population to be valid only if its amplitude exceeds a threshold. 406 This threshold is set to be half of the amplitude of the space-averaged activity at the same time 407 instance and frequency band. This conservative criterion reduces the number of spurious phase 408 synchronization indices dramatically and hence ensures the validity of the GPS.

\section{Results}

\section{Experimental Data}

412 Figure 3A illustrates the characteristic temporal evolution of EEG obtained experimentally in two 413 patients: (I) at the beginning of the surgery during light anaesthesia, (II) during deep anaesthesia 414 and (III) during the awake phase after surgery. The spectral content of the EEG during the full 415 course of surgery is shown in Fig. 3B. We note that before the start of anaesthetic administration, 416 the EEG exhibits artefactual activity in the delta-frequency range, caused by movement and muscle 417 activity. After the onset of propofol (cf. Fig.1C, red curve), oscillations in the $\beta$-band emerge and 418 gradually slow down towards the alpha-band, cf. white dashed line in Fig. 1B. In both of these 419 patients, the analgesic fentanyl was also given, and the subsequent maintenance of general 
420 anaesthesia was achieved by the volatile agent sevoflurane. After several minutes of anaesthetic 421 administration, the power peak frequency saturates in the $\alpha$-frequency band while increasing its 422 spectral power. Moreover, the initial high fentanyl concentration enhances activity in the $\delta$ 423 frequency band. At the end of surgery, the anaesthetic concentration decreases and the patient 424 emerges back to full consciousness (outside the time interval shown in Fig. 1). This emergence 425 phase is nicely reflected by an increase in the frequency of the peak power; from the $\alpha$-range back 426 to the $\beta$-frequency range, and then followed by a suppression of oscillatory power in that 427 frequency band. There is also a decrease of power in the $\delta$-frequency band. In these cases, the 428 emergence phase qualitatively resembles a backward loss of consciousness phase transition. This 429 oscillatory pattern is called the smile effect due to the spectrogram trajectory of the peak frequency. 430 Notice that in both subjects, the oscillatory transition observed is similar despite the big differences 431 in the anaesthetic concentrations. 

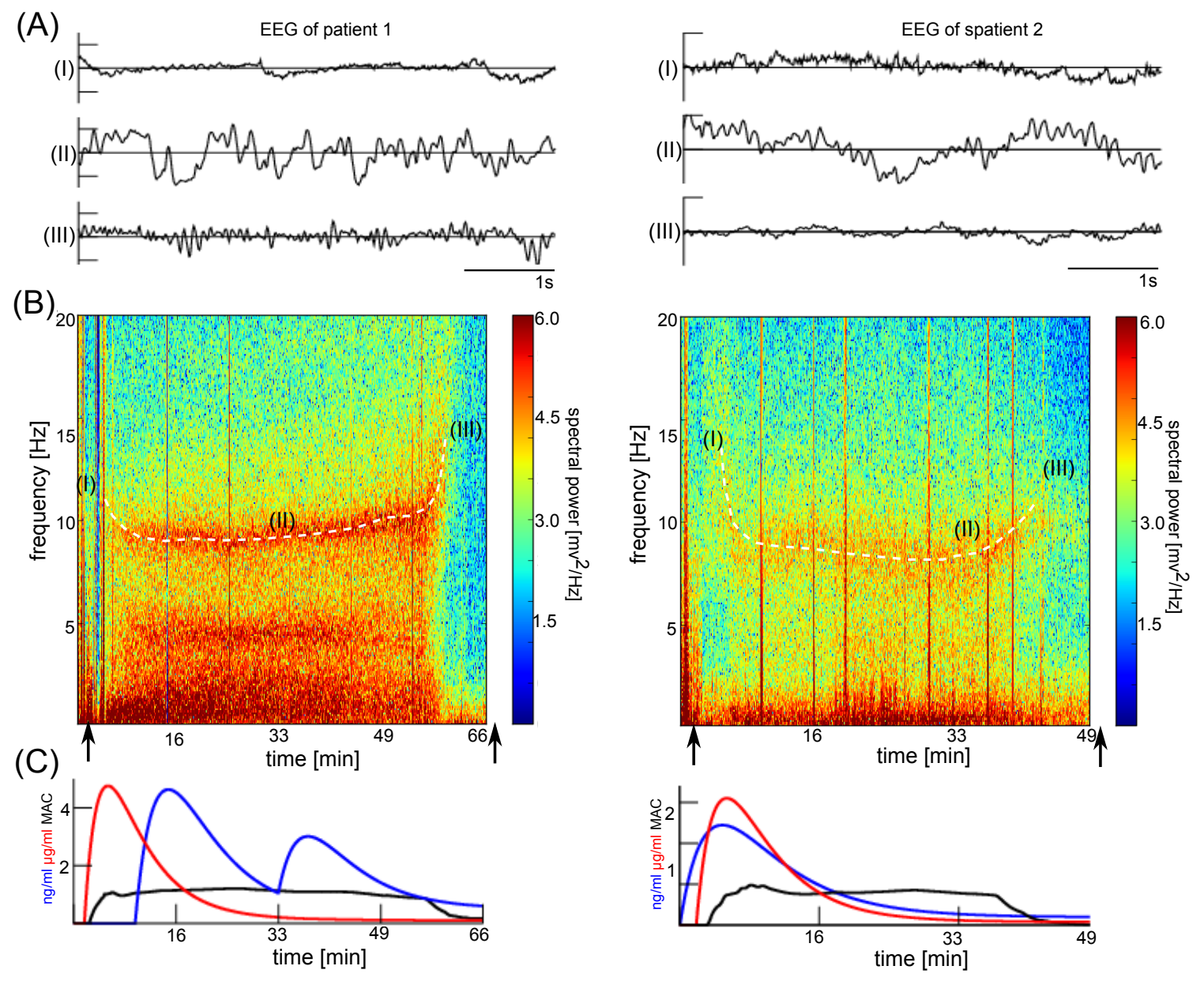

434 Figure 3: Characteristic smile effect observed in experimental frontal EEG spectral data. (A) Time 435 series of experimental frontal EEG from two patients obtained at different moments in time during 436 surgery, see (B) for encoded time points. (B) Spectral power distribution of experimental EEG data 437 from two patients from the start to the end of surgery. The white dashed line denotes the 438 frequency of maximum of $\alpha$-activity illustrating the shape of a smile. (C) Estimated effect-site 439 concentrations of the administered anaesthetic propofol (red), sevoflurane (black), and fentanyl 440 (blue), over the time course of surgery. In each panel, the left and right arrows mark the point of 441 loss of consciousness and emergence from unconsciousness in the subject, respectively. Unit of 442 fentanyl concentration is $\mathrm{ng} / \mathrm{ml}$, unit of propofol concentration is $\mu \mathrm{g} / \mathrm{ml}$ and unit of sevoflurane is 443 MAC (Minimum Alveolar Concentration). 
446 Broadband neural activity has been associated with large-scale connectivity motifs (32), whereas it 447 has been suggested that increased neural fluctuation variance is the signature of a more segregated 448 brain functional connectivity. Such segregated connectivity has been linked with increased 449 correlated brain activity and spectral power (33). To represent the broadband activity in our model 450 and in order to keep our results as generic as possible, we treated this exogenous broadband input 451 in our model as random fluctuations; since the temporal and spatial correlation structure is not 452 known.

453 In order to understand the role played by these broadband fluctuations on neural activity during 454 anaesthesia, we analysed a thalamo-cortical neural model that exhibits the joint features of generic 455 cortical and subcortical networks (Eqs. (4)). The action of anaesthetic drugs (e.g. propofol or 456 sevoflurane) was modelled in two stages: fluctuation variance reduction, and synaptic inhibition. 457 Firstly the fluctuation variance of exogenous inputs to the system ('neural noise') was decreased, cf. 458 Fig. 4A (lower panel). The evolution and spectral properties of synchronous patterns were 459 recorded for each epoch. This showed an induction of $\beta$-band activity followed by a generic 460 deceleration of oscillatory activity, transitioning from the $\beta$ band to the $\alpha$ band (Fig. 4A). An 461 increase of the fluctuation level - as would occur when the concentration of anaesthetic drugs 462 decrease - inverts the characteristic spectral features; giving rise to a spectrogram pattern like the 463 characteristic anaesthetic "smile" in Fig. 2. The second modelled effect of anaesthetic drugs was to 464 follow much previous literature and cause an increase in inhibitory synaptic gain. Figure 2B reveals 465 that additional synaptic anaesthetic action does not qualitatively change the simulated EEG. 

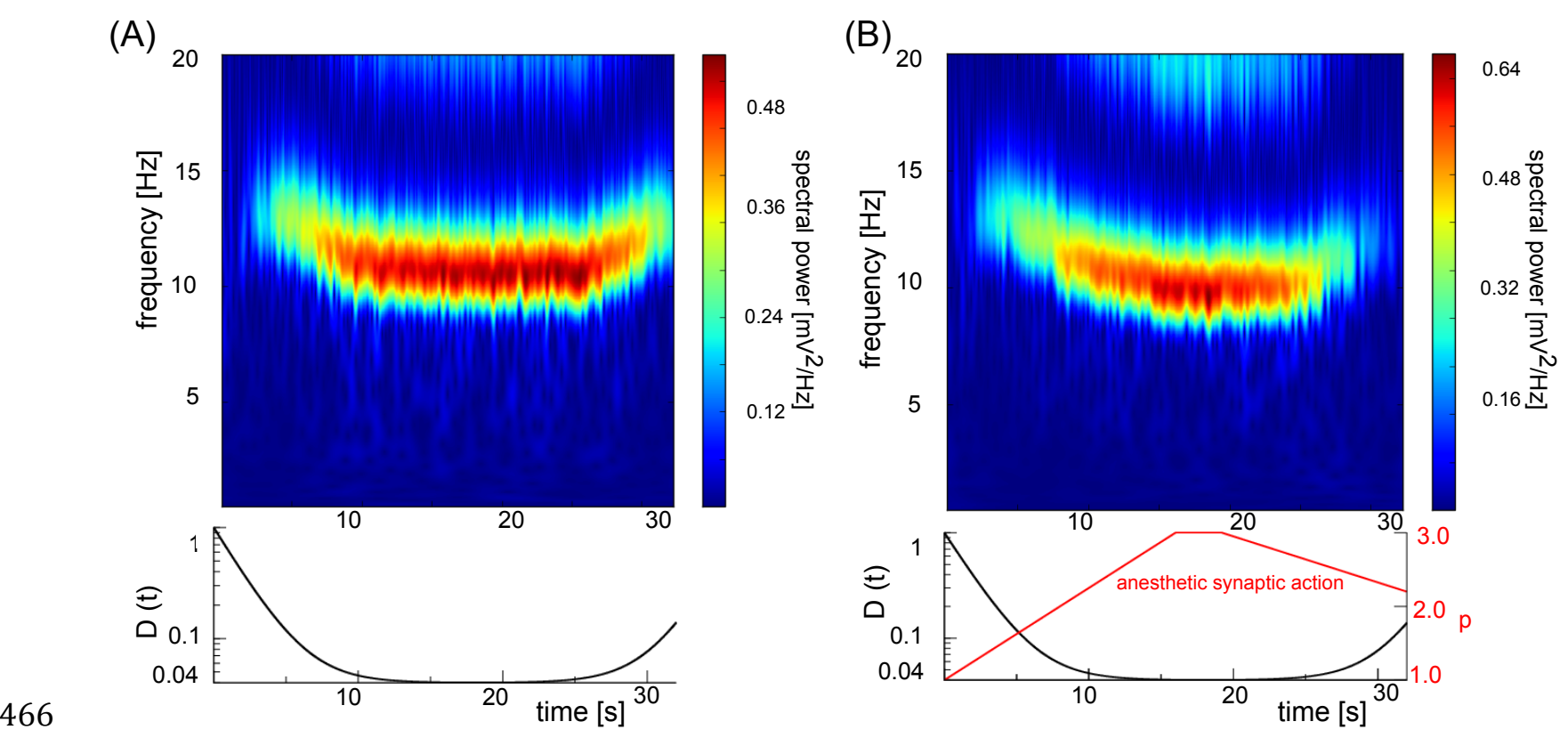

467 Figure 4: Numerical simulation of frontal EEG based on the frontal spiking neuron network model.

468 (A) global drive by random fluctuations (B) global drive by random fluctuations with additional 469 anaesthetic synaptic action. Spectral power of EEG (top panel) is given together with time470 dependent fluctuation variance (bottom panel) .

472 The latter results show that weakening fluctuations induce oscillations in the cortical dendritic 473 activity. In parallel spikes synchronize rhythmically both the cortex and thalamus. Figure 5 474 illustrates this finding in the spiking activity of a subset of neurons in the spiking network model in 475 the absence of anaesthetic synaptic action (A). Initially, at high fluctuation variance (left panels), 476 spiking is irregular and asynchronous but becomes more regular after some time when fluctuation 477 variance decreases (right panels). At minimum fluctuation variance, the cortical E-cells burst with 478 an inter-burst rhythm of $9 \mathrm{~Hz}$. The thalamic LGN cells burst at a rate of $4.5 \mathrm{~Hz}$, i.e. half of the burst 479 firing rate of cortical neurons, and they synchronize with every second burst of cortical neurons. 480 Simulated EEG weakly synchronizes with cortical E cell spiking at large fluctuation variance, 481 whereas it strongly synchronizes with E cell spikes at low fluctuation variance. Adding anaesthetic 
synaptic action (B) aligns few cortical spike trains in the E-population at low fluctuation variance.

483 The aligned spike trains exhibit sparse burst activity; much less synchronous than under absence of 484 anaesthetic synaptic action. LGN cells cease to spike regularly and almost stop spiking due to the increased synaptic inhibition. Similar to (A), EEG still synchronizes well with E cell spiking activity.

(A)
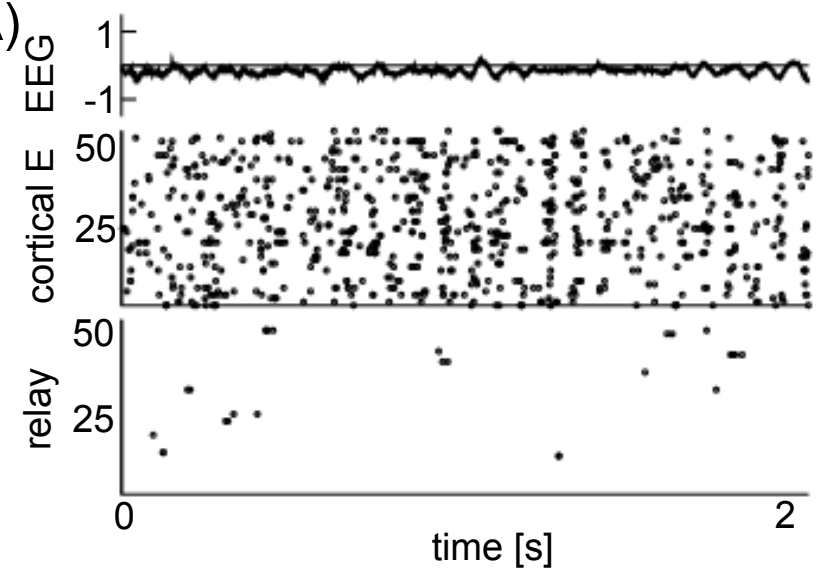

(B)
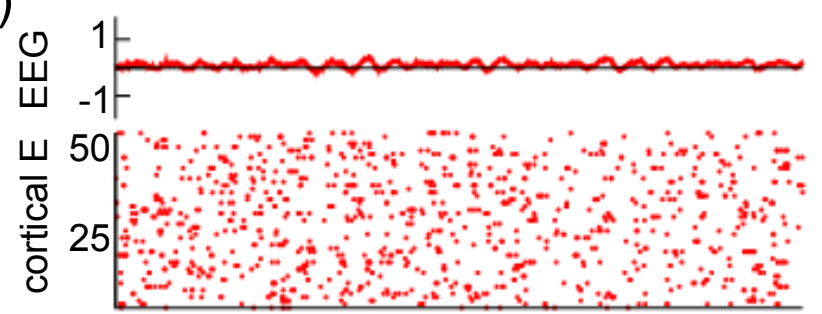

486

487

488

489

490

491

492 neurons. 'relay' = thalamo-cortical relay neurons in the LGN.
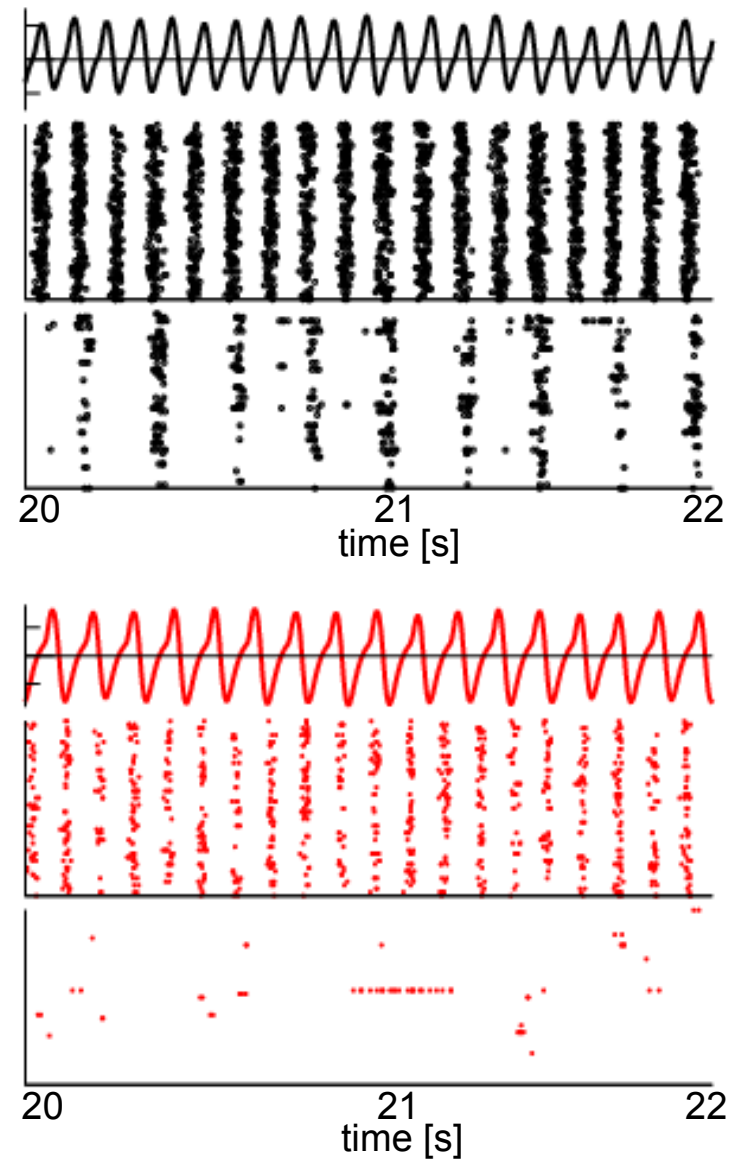

Figure 5: Increasing regularity of frontal EEG and spiking activity in frontal cortex and thalamus with increasing time and decreasing amplitude of random fluctuations. (A) global drive by random fluctuations (B) global drive by random fluctuations with additional anaesthetic synaptic action. The fluctuation level decreases according to Fig. 2, bottom panel. For better visualization, panels show spike events of 50 neurons only arbitrarily chosen in each neural structure. 'E' = excitatory 
494 Encephalographic activity represents the collective activity of neurons populating superficial 495 cortical networks. To understand better the fluctuation-induced spectral change observed in Figs. 3 496 and 4, we performed a mean-field analysis of a reduced cortical spiking network subjected to 497 additive noise.

(A)

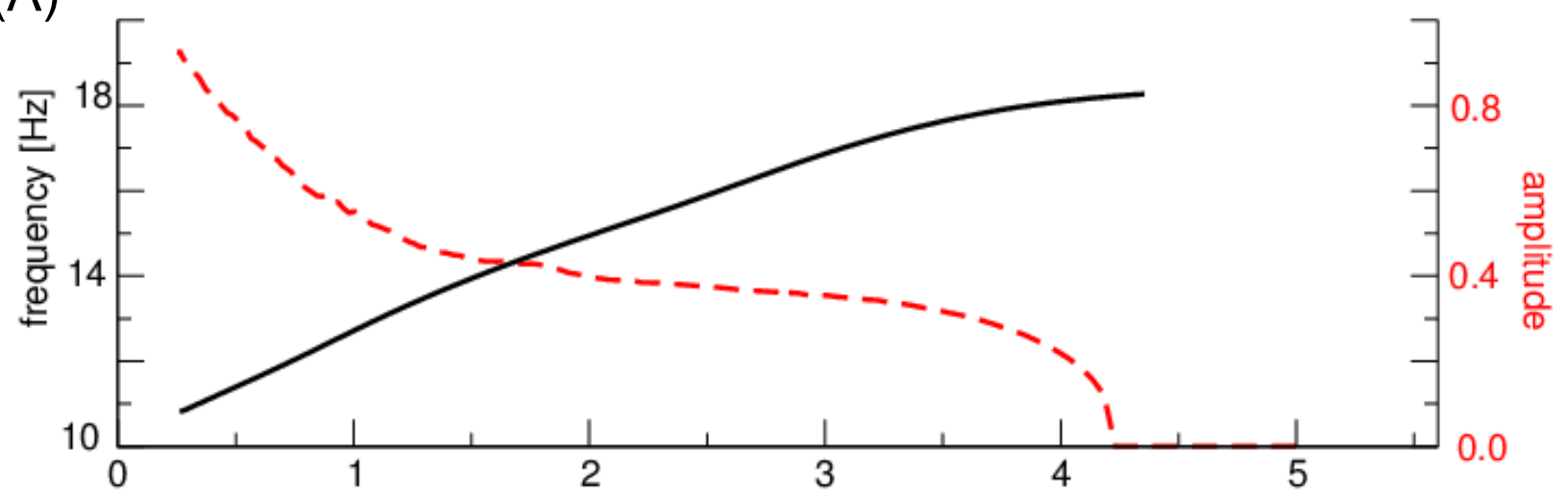

(B) noise level D
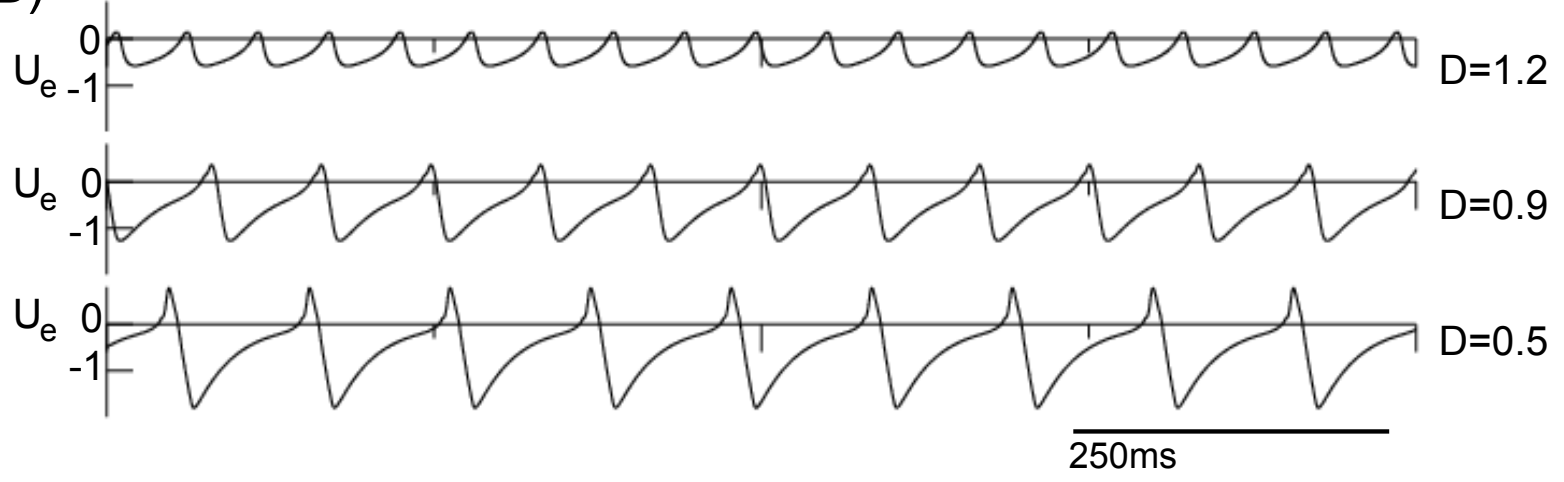

499

500

501

502

503

504 505

Figure 6: Random fluctuation-induced smile effect originates from an underlying oscillatory instability (Hopf bifurcation). (A) The amplitude (red) and frequency (black) of deviations $\mathrm{U}_{\mathrm{e}}(\mathrm{t}$ ) from the systems stationary state are computed numerically based on the mean-field model, see Eqs. (5). The large value of D compared to Fig. 4 and 5 compensates for the missing fluctuations input from the thalamus - which is included in the full spiking model. (B) Simulated mean-field EEG subjected to different noise levels. 
We find that, at high fluctuation levels ( $\mathrm{D}>4.2)$, the system does not oscillate and deviations from

507 the stationary state have zero amplitude, cf. Fig. 6. Decreasing the fluctuation level induces loss of 508 stability (Hopf bifurcation) resulting in increasing amplitude, initially in the $\beta$-band, but then 509 slowing to the $\alpha$-band. A noise-induced instability has been found recently in more simple neural 510 models (39). Hence additive fluctuations alone are able a produce a shift in the network stability

511 which is reflected by the observed changes in the system frequency and oscillation amplitude. This 512 explains the frequency and magnitude tuning by modified fluctuation variance in the full model 513 observed in Figs. 3-5.

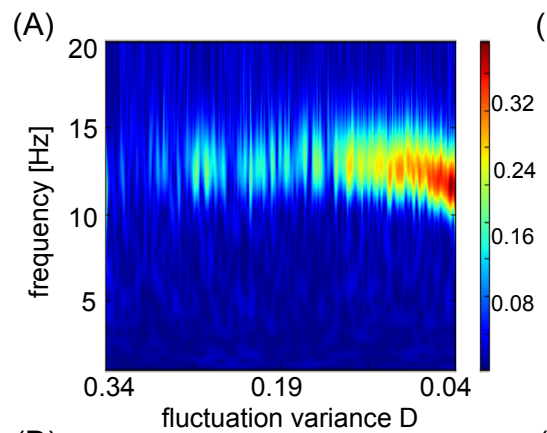

(D)

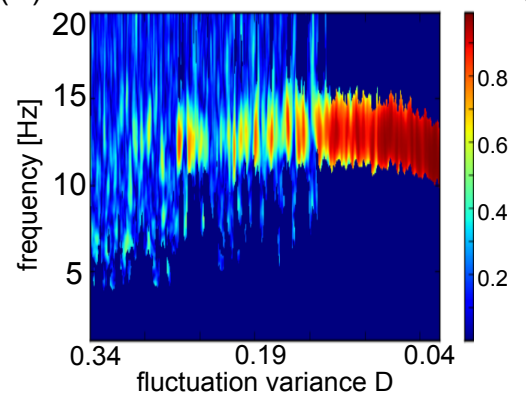

(B)

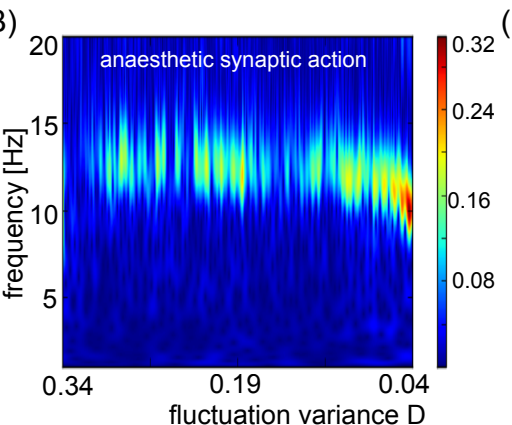

(E)

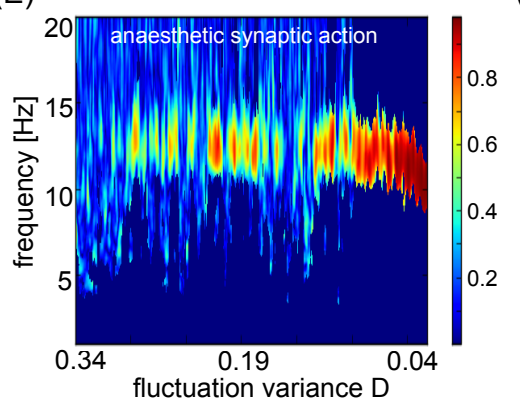

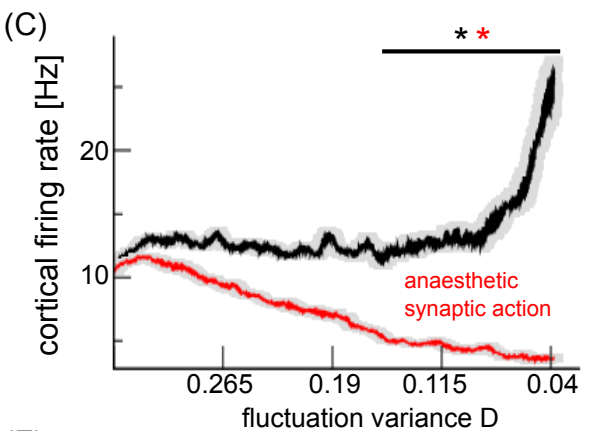

(F)

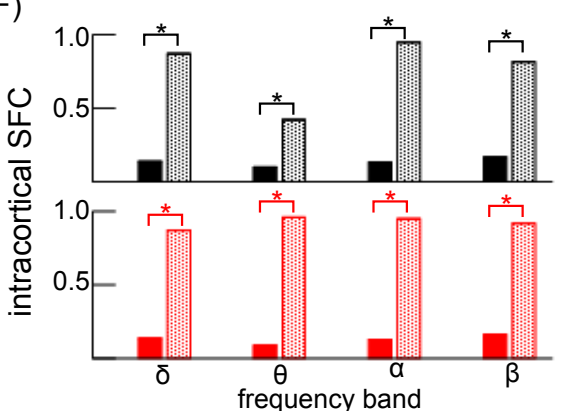

516 Figure 7: Spectral power, firing rate and synchronization in the frontal cortex with decreased

517 fluctuation variance. (A) Power spectrum without anaesthetic synaptic action. (B) Power spectrum

518 with anaesthetic synaptic action. (C) Mean population firing rate of E-cells. Error bars (grey) denote

519 standard errors, firing rates below the horizontal bar are significantly different to firing rates at

520 largest fluctuation variance ( $\mathrm{p}<0.001$, Kruskal-Wallis test) (D) Global phase synchrony of simulated

521 LFPs without anaesthetic synaptic action. (E) Global phase synchrony of simulated LFPs with 
522 anaesthetic synaptic action. (F) Intra-area spike-field coherence between E-cells, error bars denote

523 standard errors. For all panels, the fluctuation variance D varies according to Fig.4, bottom panel.

\section{Connectivity in the frontal network model}

525 To better illustrate intra-cortical dynamics, Fig. 7 shows the spectral power (A) and the population

526 firing rate (C, black) of dendritic activity in the modelled cortical excitatory neuronal population

527 without synaptic anaesthetic action. The power and the firing rate $(\mathrm{p}<0.001)$ increases as the

528 fluctuation variance decreases. The corresponding intra-area phase locking index of dendritic

529 activity (D) between excitatory cells in the cortical area reveals a monotonic phase synchronization

530 enhancement as fluctuation variance decreases. Similarly, weakening fluctuations enhances the

531 intra-area SFC (F, black) in all frequency bands dramatically. This increase of intra-area SFC

532 coincides with increasing phase synchrony (see panel D) when the peak of maximum spectral EEG

533 power (panel a) shifts from the $\beta$-band to the $\alpha$-band.

534 Considering anaesthetic action on the inhibitory synaptic gain in addition, the power surge (B),

535 phase synchrony (E) in the $\beta$-and $\alpha$-band and SFC (F, red) are retained; but we now see a more

536 realistic statistically significant $(\mathrm{p}<0.001)$ decrease in the population firing rate $(\mathrm{C}$, red $)$. This

537 decrease of firing rate is in line with experimental findings in the cortex in the presence of various

538 anaesthetic agents (21).

540 The cross-spike-field coherence (CSFC) between different populations behaves differently.

541 Weakening fluctuations markedly diminishes the coherence between cortical excitatory cells and

542 thalamic cells as seen in Fig. 8. In the absence of inhibitory synaptic anaesthetic action, low

543 fluctuation variance makes the CSFC vanish in all frequency bands except for the $\alpha$-band where it

544 increases in the $\alpha$-band. The addition of inhibitory synaptic anaesthetic action to the model 
545 eliminates the spike-field coherence in all frequency bands. Hence cortex and thalamus functionally 546 decouple.
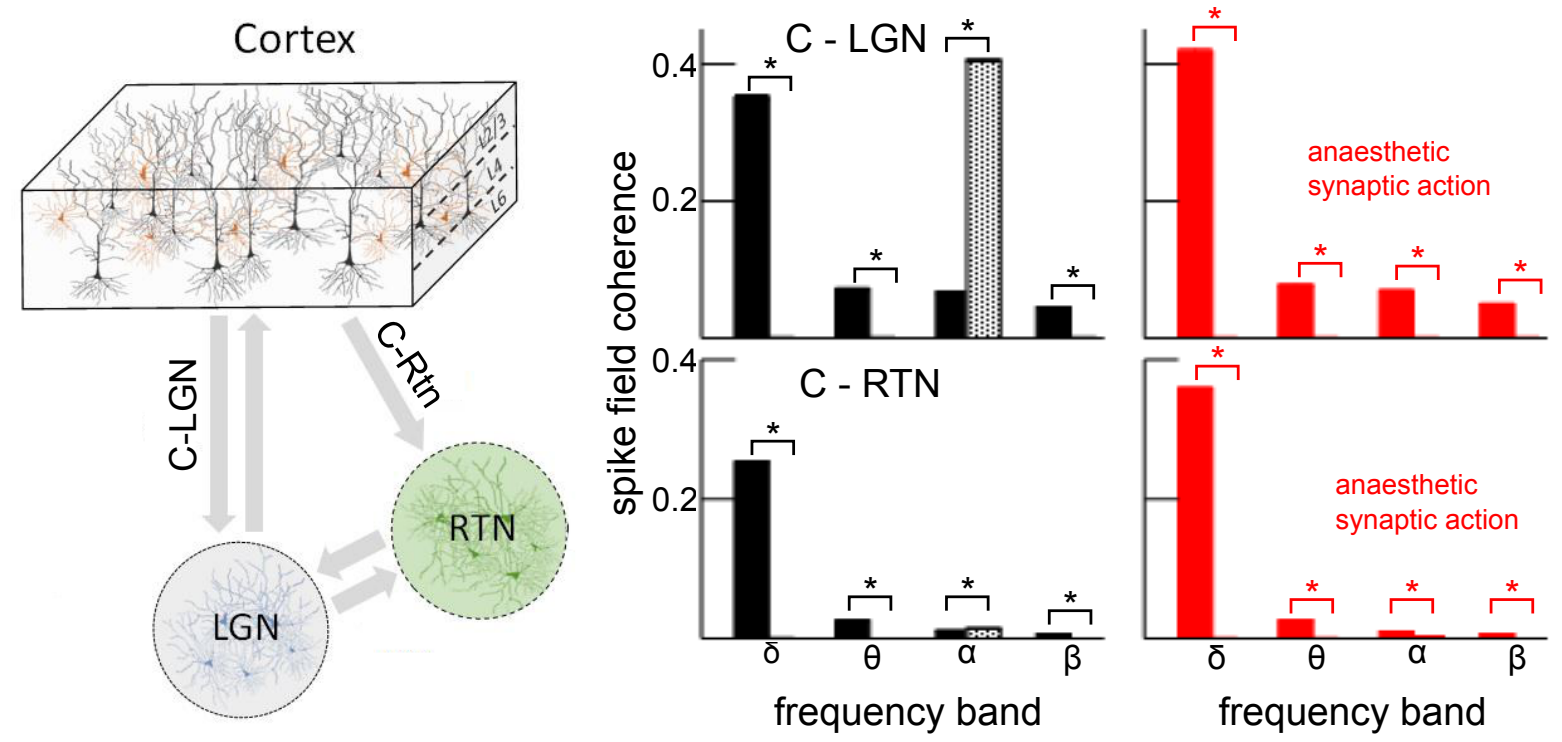

Figure 8: Frontal cortex and the thalamic structures decouple for weak random fluctuations. Panels on right hand side show the cross spike-field coherence between cortex and LGN (C-LGN) and between the cortex and the thalamic reticular area (C-RTN) subject to the fluctuation intensity in the absence (black) and presence (red) of anaesthetic synaptic action. Error bars denote standard errors, $\mathrm{p}<0.0001$ (Kruskal-Wallis test)

557 Figure $9(\mathrm{~A})$ shows strong activity in the $\alpha$-band in the beginning (large fluctuation variance) that

558 drops rapidly after some time (small fluctuation variance). This transition is in good accordance to 559 experimental data $(16,35)$. Since this posterior $\alpha$-activity drops while the anterior $\alpha$-activity 

emerges (see previous sections), this effect is called anteriorization (50). The occipital $\alpha$-rhythm

561 emerges from a highly synchronous E-population (Fig.9(B)) that retains its phase synchrony over 562 time, i.e. for all fluctuation variances. The high initial $\alpha$-power is accompanied by synchronous 563 spiking in the E-population (at large fluctuation variance), whereas spiking stops at large times 564 (low fluctuation), cf. Fig. 9(C). At a first glance, this seems to contradict the persistent strong phase 565 synchrony between the cells. However, thalamic LGN cells spike highly synchronous persistently 566 over time at a frequency of $\sim 10 \mathrm{~Hz}$ driving the E population. This thalamo-cortical drive ensures the 567 strong E-cell phase synchrony.
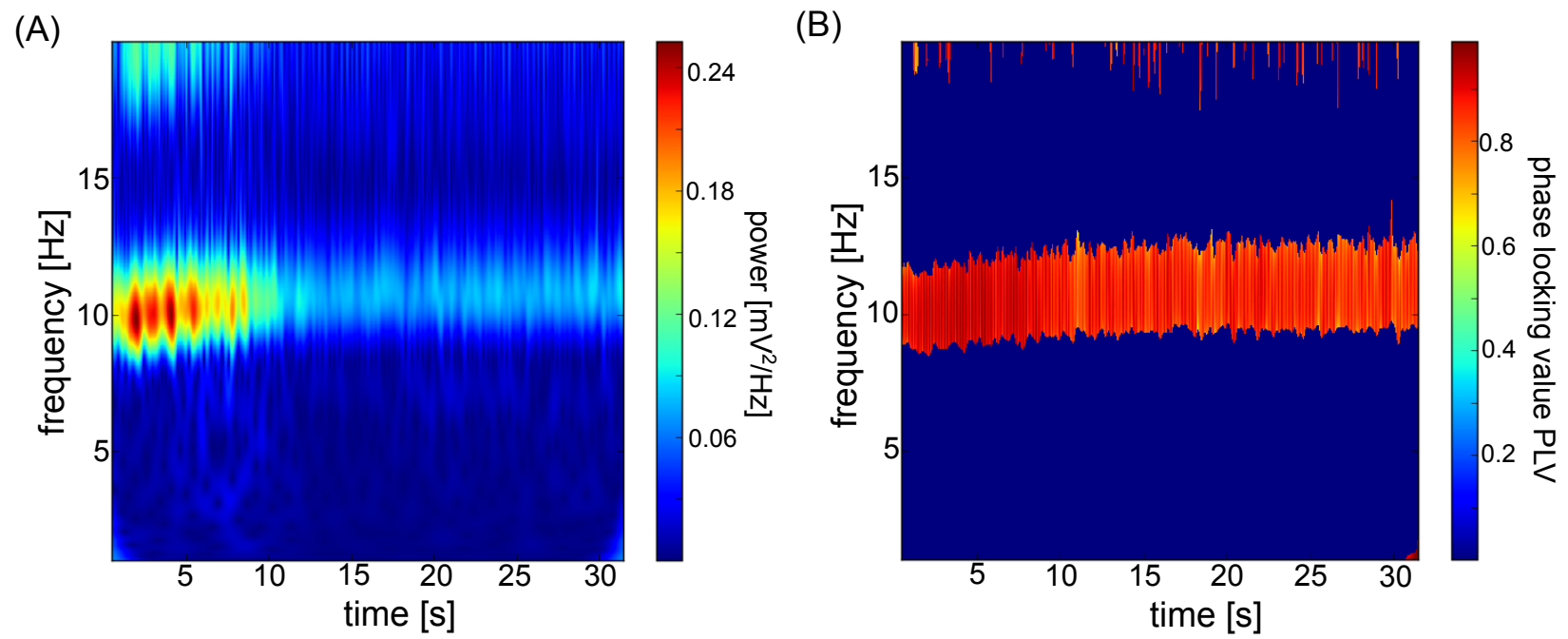

(C)
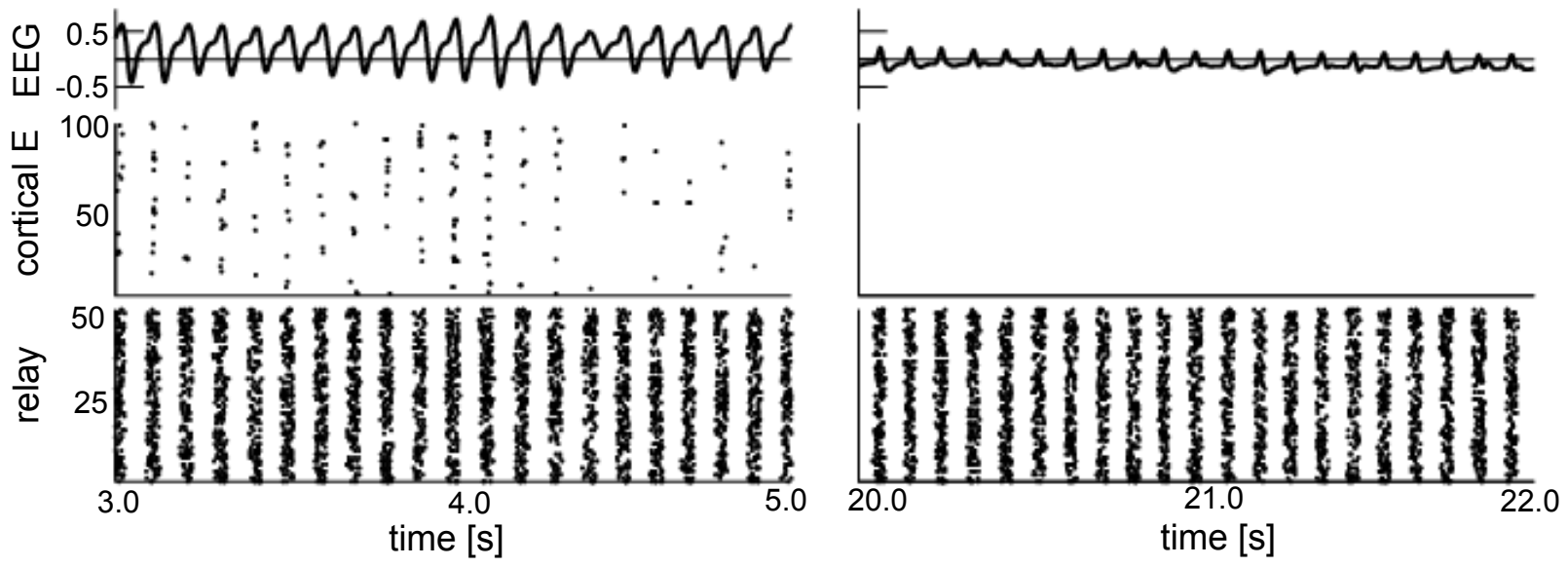
569 Figure 9: Occipital $\alpha$-activity drops with increasing fluctuation variance while LGN activity is 570 retained. (A) Time-frequency distribution of numerically simulated EEG. (B) Global phase 571 synchrony in the E population. (C) EEG time series (top panel), spiking activity in a subset of 100 572 cortical E cells (centre panel) and in a subset of 50 LGN cells (bottom panel).

573 To understand better network interactions under anaesthesia, it is insightful to take a closer look at 574 inter-area connectivity. Figure 10 reveals the fragmentation of occipital cortex and the thalamic 575 areas PGN and RTN.

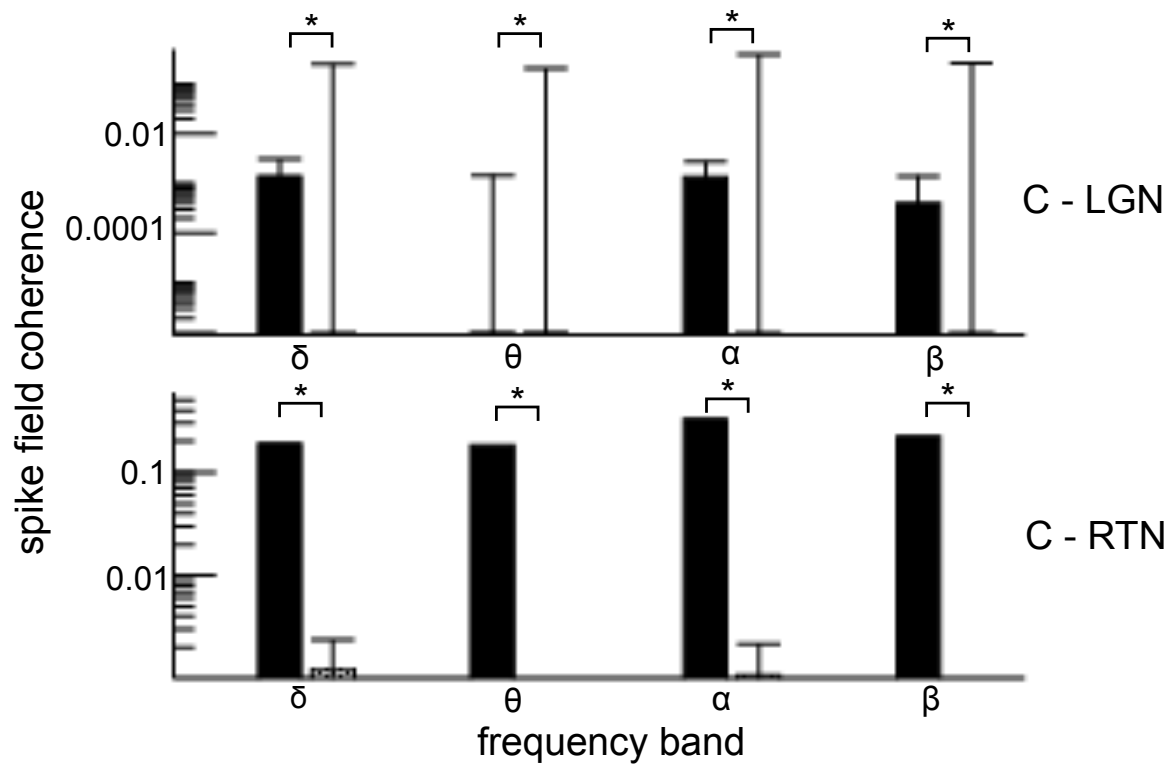

577 Figure 10: Inter-area connectivity between occipital cortex and the thalamic areas in different 578 frequency bands. The solid and dotted bars denote the median spike field coherence (SFC) at high $579(\mathrm{D}=1.0)$ and low $(\mathrm{D}=0.01)$ fluctuation variance, vertical bars denote the standard error. All SFC 580 distributions in all frequency bands at high and low fluctuation variances are significantly different $581 \quad(\mathrm{p}<0.00001)$ 
585 The model we propose here is able to reproduce a number of observed characteristics of 586 propofol/sevoflurane anaesthesia. It describes the characteristic changes in EEG power and 587 frequency in the $\beta$ - and $\alpha$ - frequency range, the increase in local neuronal intra-population 588 synchrony combined with loss of distant neuronal inter-population synchrony, and the decrease in 589 neuronal firing rate.

591 Previous experimental studies on the effect of general anaesthetics have shown an initial induction 592 of enhanced neural activity in the $\beta$ frequency range with a subsequent peak frequency decrease to 593 the $\alpha$-frequency band - the so-called smile effect or travelling peak mechanism (34) - with 594 changing anaesthetic concentrations $(13,16,17,35)$. Examples of this phenomenon are shown in 595 Figure 3. During this transition subjects typically lose consciousness. In recent years, many studies 596 have focussed on the reverse transition from unconsciousness back to consciousness and usually 597 revealed a reversal of these spectral characteristics $(15,35)$.

599 Our results show that time-frequency characteristics in the $\beta$ and $\alpha$ frequency range can be 600 explained by a state-dependent change in broadband neural activity. The onset of $\beta$-activity and the 601 subsequent frequency decrease into the $\alpha$-range can be explained by a transition from a stable to an 602 unstable oscillatory neural state, cf. Fig. 6.

603 The intra-population phase synchrony of the model EEG increases with decreasing fluctuation 604 intensity (Fig. 6) both in the absence and presence of anaesthetic inhibitory synaptic effects. This is 605 in line with experimental findings of enhanced EEG-coherence in the $\alpha$ band at large dose of 606 propofol (16).

607

608 Unlike the EEG patterns - which seem to be largely driven by levels of neural fluctuations - 
neuronal ensemble spiking activity is more sensitive to the anaesthetic enhancement of inhibitory

610 synaptic effects. When the input fluctuations weaken cortical neurons start bursting synchronously,

611 but their synchrony weakens with increased anaesthetic inhibitory synaptic action (Figs. 5 and 7).

612 This is also reflected in their population firing rate which first decreases, but subsequently

613 increases as fluctuation variance is further decreased (if no anaesthetic synaptic action is included

614 in the model). Conversely the population firing rate decreases monotonically if increasing

615 anaesthetic synaptic inhibition is added to the model. These different effects indicate that it is the

616 relative level of fluctuation variance and anaesthetic inhibitory synaptic action that determines the

617 population firing rate. This finding corroborates the results of a previous experimental study (17)

618 which showed a wide range of firing rates in the prefrontal cortex after LOC in different patients -

619 both below and beyond the firing rate measured before LOC.

620

621 Relay cells in the LGN exhibit synchronous bursts in the absence of anaesthetic synaptic action. This

622 is in line with the burst activity observed experimentally in thalamic LGN neurons of un623 anaesthetized animals (36) and in non-REM sleep stages (37). Such burst-like activity in the LGN 624 has been postulated to block meaningful information transfer to the cortex (38). However under 625 anaesthetic synaptic action, LGN cells reduce their firing activity dramatically (Figs. 5 and 7).

627 One of the strengths of our approach is that the phenomenon we describe is fundamentally driven 628 by changes in pre-synaptic firing rates while assuming a generic neuron model. Hence the results 629 are robust to variations in neuron model parameters and does not require fine tuning. The 630 population dynamics predicted by our model are diverse and comprise both irregular firing and 631 bursting, originating from circuit interaction. This can be generated and altered in many ways, and 632 thus does not depend specifically on the neuron model we have chosen; or indeed the specific 633 neuronal effects of each different anaesthetic drug. 
635 Link between anaesthetic action and intrinsic fluctuation variance

637 Using human data, and building on the well-known experimental finding that most anaesthetics 638 affect single neurons and populations by specific receptor and ion channel action (21), our work 639 departs from previous modelling studies by introducing the notion that a generic decrease in neural 640 fluctuations in the global brain network originating in the ascending arousal system may tune the 641 transitions observed under anaesthesia. A converging line of evidence indicates that anaesthesia 642 enhances the overall inhibition in the brain stem, which triggers a reduction in random input to the 643 cortex and the thalamus (39). In the current debate on the question of whether general anaesthetics 644 act primarily at cortical or subcortical levels, our model would lend support to the importance of 645 subcortical actions. It would also suggest that a progressive frontal increase in $\alpha$-frequency as 646 anaesthesia concentration decreases at the end of surgery, could be a biomarker of gradual increase 647 in AAS activity, that is associated with a good post-operative recovery (40). This is also supported 648 by our finding that decreasing AAS induces a drop of $\alpha$-power in occipital areas in very good 649 accordance to experimental data $(16,35)$. Conversely, failure to show an increase in peak frequency 650 before waking might be an indication that the subcortical structures have not activated in the 651 correct order and are unprepared for the arousal - and hence is associated with more 652 postoperative delirium.

\section{Intra- and inter-area functional connectivity}

656 Large random fluctuations in a system result in broadband neural activity, which is associated with 657 large-scale connectivity motifs involving a plurality of time scales (32). This allows brain circuits to 658 explore their dynamic repertoire (41). Figure 7 reveals strong increases in phase synchrony of 
electric potentials in the $\alpha$-band within the cortical population at low fluctuation variance (i.e.

660 during anaesthesia); which agrees with experimental studies demonstrating strong synchronized

661 frontal $\alpha$-activity close to the point of loss of consciousness $(16,35)$ and increased regional

662 synchrony under sedation $(52,53)$. The intra-area neuronal spike-field coherence quantifies

663 functional connectivity based on the synchrony between spikes and co-localized electric potentials.

664 Weak input fluctuations yield high coherence between cortical cells both with and without the

665 inclusion of anaesthetic synaptic action in the model.

666

667 Considering inter-area connectivity, the cross-spike-field coherence between cortical and thalamic

668 areas vanishes at low fluctuation variances with anaesthetic synaptic action, cf. Fig. 8, indicating a

669 functional isolation of cortex from thalamus during anaesthesia. Such a decoupling between cortical

670 and subcortical populations has been hypothesized theoretically (42) and adds to a growing body

671 of evidence in sleep $(42)$ and in anaesthesia $(17,43)$. Our results thus supports that de-noising the

672 brain functionally decouples it and hence renders it more regular. This aligns with recent

673 experimental results (44): studying local field potentials in ferrets under anaesthesia, the authors

674 found reduced information transfer between prefrontal cortex and visual cortex with increased

675 anaesthetic concentration. This decrease was associated with augmented active information

676 storage (AIS) and reduced differential entropy (DE) in each area. Large AIS represents much stored

677 information while low DE reflects less available information to be transferred. In this information

678 theoretic framework, the enhanced intra-area functional connectivity observed experimentally in

679 EEG and found in our model as augmented phase synchrony represents increased AIS and hence

680 reduces the information that can be transferred. Moreover, a previous functional magnetic

681 resonance imaging study (45) found a reduced repertoire of internal brain states under propofol

682 anaesthesia. This is in line with the increased AIS and with suggested role of broadband neural

683 activity in exploring the dynamical repertoire of neural networks (41). In addition, these results are 
684 in agreement with the reported fact that random fluctuations - or neural variability - is beneficial to

685 information processing and, consequently, less variability reduces the information flow between

686 neural structures (46). Taken together, our work complements these studies by showing that

687 weakening fluctuations not only hampers information flow between neural populations but 688 consequently splits up interconnected networks.

689 In sum, our results show that weak fluctuations in the brain circuit cause a nonlinear oscillatory 690 instability, yielding a local intra-area synchrony in the $\alpha$ frequency band. This intra-area synchrony 691 disrupts synchrony between cortex and thalamus and hence fragments the various populations 692 involved. Since the LGN is the relay structure of information flow, we conclude that its synchrony 693 loss to the cortex contributes primarily to the loss of consciousness.

\section{Perspectives of the model}

697 Other studies on the origin of sleep-wake transitions have highlighted the importance of the AAS 698 (47) controlling the brain excitation level. Various specific aminergic and cholinergic nuclei of the 699 AAS originate in the brainstem and innervate both the cortex and the thalamus, activating LGN 700 neurons as well as the reticular nuclei. Our proposed model takes into account noisy thalamic and 701 cortical input assuming that broadband neural activity in the thalamo-cortical system is controlled 702 by the brainstem; but it could equivalently be considered as coming from other uncorrelated 703 distant cortical sources. This points to a synergistic interaction between the increased GABA effects 704 of anaesthetic drugs and the decrease in neuronal fluctuations. The increased hyperpolarisation in 705 distant brain structures, caused by the GABAergic actions, will themselves cause decreased 706 fluctuations in the input to the region of brain under consideration. Thus the observed changes in 707 EEG pattern might be secondary to GABAergic actions at a distant region. As an aside, the model 708 proposed fits well with the known effects of ketamine on the EEG. This drug causes an increase in 
alpha frequency and then loss of $\alpha$-power, presumably the result of its known effect of increasing

710 brain stem noise input to the thalamus and cerebral cortex. However the causes of ketamine-

711 induced cortical segmentation are not explained by our model, and remain an enigma.

712

713 The proposed spiking population model and the derived mean-field model consider homogeneous

714 spatial neural populations. This homogeneity approximation is valid for describing EEG spectra

715 (33). More detailed experimental studies focussing on the spatio-temporal evolution of Local Field

716 Potentials in rats under isoflurane anaesthesia have discovered a sequence of meta-stable neural

717 states during the emergence phase (48). These meta-stable states are spatial patterns that are

718 activated in a specific temporal sequence. Since meta-stable states require specific non-

719 homogeneous network interactions (49) while the present model network is homogeneous, such

720 meta-stable states are not solutions of our model, but may be elements of future work.

721

722 Previous network studies of coupled individual neurons have also reproduced qualitatively frontal 723 and EEG (8) and anteriorisation (50) by considering anaesthetic effects in individual neurons. Even

724 neural mass network models have been shown to reproduce both frontal EEG spectral features and 725 anteriorisation (51). To our best knowledge, the present study is one of the first to involve the AAS

726 demonstrating its importance. We have considered both frontal and occipital cortical areas, but

727 have neglected other brain areas that are known to exhibit diverse anaesthetic impact. Examples

728 are the parietal cortex and the areas of the default mode network that respond differently at

729 different anaesthetic depths $(52,54)$. Moreover the work does not consider deep anaesthesia where

730 other characteristic features emerge such as burst suppression, slow frequencies $(<1 \mathrm{~Hz})$, an intra-

731 area fragmentation (52) or isoelectric activity (55).

732

733 As a conclusion, the present work proposes that anaesthetic action modifies the global regularity of 
734 intrinsic neural activity. Increasing anaesthetic concentration decreases broadband fluctuations, 735 enhances the coherence of intra-area neural activity, and induces a functional decoupling of the

736 thalamo-cortical circuit. The parallel increase in intra-area functional connectivity accompanied by

737 a reduction of functional connectivity between network areas is a direct consequence of amplified

738 correlations in neural activity.

739

740 Acknowledgements

741 This work has been supported by the Natural Sciences and Engineering Research Council of Canada 742 (JL).

743

744 Contributions

745 All authors designed the study, analysed the data, and wrote the paper.

746

747 Competing interests

748 We state that there are no competing interests.

749 Declaration of interests:

750 None.

751

752

753 References 
754 1. Grasshoff C, Drexler B, Rudolph U and Antkowiak B (2006). Anaesthetic drugs: linking molecular 755 actions to clinical effects. Curr. Pharm. Des. 12(28) : 3665-3679

756 2. Alkire MT, Hudetz AG and Tononi G (2008). Consciousness and Anesthesia. Science 322 : 876 757 880. doi :10.1126/science. 1149213

758 3. Sellers K, Bennett D, Hutt A, Williams J and Frohlich J (2015). Awake versus Anesthetized: Layer759 Specific Sensory Processing in Visual Cortex and Functional Connectivity between Cortical Areas, J. $760 \quad$ Neurophysiol. 113(10): 3798-815.

761 4. Barttfeld P, Uhrig L, Sitt JD, Sigman M, Jarraya B and Dehaene S (2015) Signature of 762 consciousness in the dynamics of resting-state brain activity, Proc. Natl. Aca. Sci. USA 112(3) : 887763 889. Doi : 10.1073/pnas.1418031112

764 5. Schultz A, Siedenberg M, Grouven U, Kneif T and Schultz B (2008). Comparison of Narcotrend 765 Index, Bispectral Index, spectral and entropy parameters during induction of propofol-remifentanil 766 anaesthesia, J. Clin. Monit. Comput. 22 :103-111.

767 6. Hight D, Voss LJ, Garcia PS and Sleigh J (2017). Changes in Alpha Frequency and Power of the 768 Electroencephalogram during Volatile-Based General Anesthesia. Front. Syst. Neurosci. 11 :36. doi :

$769 \quad 10.3389 /$ fnsys.2017.00036

770 7. McCarthy MM, Brown EN and Kopell N (2008). Potential Network Mechanisms Mediating 771 Electroencephalographic Beta Rhythm Changes during Propofol-Induced Paradoxical Excitation. J. $772 \quad$ Neurosci. 28(50) : 13488-13504.

773 8. Ching S, Cimenser A, Purdon PL, Brown EN and Kopell NJ (2010). Thalamocortical model for a 774 propofol-induced-rhythm associated with loss of consciousness. Proc. Natl. Acad. Sci. USA 107(52): $775 \quad 22665-22670$. 
776 9. Bojak I, Day HC and Liley DTJ (2013). Ketamine, propofol, and the EEG: a neural field analysis of

777 HCN1-mediated interactions. Front. Comput. Neurosci. 7: 22

778 10. Hashemi M, Hutt A and Sleigh S (2014). Anesthetic action on extra-synaptic receptors: effects in 779 neural population models of EEG activity. Front. Syst. Neurosci. 8, 232. doi: $780 \quad 10.3389 /$ fnsys.2014.00232

781 11. Hendrickx JF, Eger EI, Sonner JM and Shafer SL (2008). Is synergy the rule? A review of 782 anesthetic interactions producing hypnosis and immobility. Anesth. Analg. 107(2):494-506 . doi:

783 10.1213/ane.0b013e31817b859e

784 12. Steriade M, Amzica F, Contreras D (1994). Cortical and thalamic cellular correlates of 785 electroencephalographic burst-suppression. Electroencephalogr. Clin. Neurophysiol. 90:1-16.

786 13. Sellers KK, Bennett DV, Hutt A and Fröhlich F (2013). Anesthesia differentially modulates 787 spontaneous network dynamics by cortical area and layer. J. Neurophysiol 110:2739-2751. 788 doi:10.1152/jn.00404.2013

789 14. Hudetz AG, Liu X, Pillay S, Boly M, Tononi G (2016). Propofol anesthesia reduces Lempel-Ziv 790 complexity of spontaneous brain activity in rats. Neurosci Lett.628:132-5. doi:

$791 \quad 10.1016 /$ j.neulet.2016.06.017

792 15. Vizuete JA, Pillay S, Ropella KM and Hudetz AH (2014). Graded Defragmentation of cortical 793 neuronal firing during recovery of consciousness in rats. Neuroscience 275: 340-351. doi : 794 10.1016/j.neuroscience.2014.06.018

795 16. Cimenser A, Purdon PL, Pierce ET, Walsh JL, Salazar-Gomez AF, Harrell PG, Tavares-Stoeckel C, 796 Habeeb K and Brown EM (2011) Tracking brain states under general anesthesia by using global 797 coherence analysis , Proc. Natl. Aca. Sci. USA 108(21) : 8832-8837. doi: 10.1073/pnas.1017041108 
17. Lewis LD, Weiner VS, Mukamel EA, Donoghue JA, Eskandar EN, Madsen JR, Anderson WS

799 Hochberg LR, Cash SS, Brown EN and Purdon PL (2012) Rapid fragmentation of neuronal networks

800 at the onset of propofol-induced unconsciousness. Proc. Natl. Acad. Sci. USA109(49):19891-19892.

801 doi: 10.1073/pnas.1210907109

802 18. Alkire MT and Miller J (2005). General anesthesia and the neural correlates of consciousness.

803 Prog Brain Res. 150:229-44.

804 19. Brown EN, Lydic R and Schiff ND (2010) General Anesthesia, Sleep and Coma. N. Engl. J. Med.

805 363: 2638-2650.

806 20. Saper CB, Scammell TE, Lu J. (2005) Hypo-thalamic regulation of sleep and circadian rhythms.

$807 \quad$ Nature 437:1257-63

808 21. Franks NP (2008) General anaesthesia: from molecular targets to neuronal pathways of sleep

809 and arousal. Nat Rev Neurosci_.9(5):370-86. doi: 10.1038/nrn2372.

810 22. Kopanitsa MV (1997). Extrasynaptic receptors of neurotransmitters: distribution, mechanisms

811 of activation, and physiological role. Neurophysiol. 29 : 448-458.

812 23. Mitchell SJ and Silver RA (2003) Shunting inhibition modulates neuronal gain during synaptic

813 excitation. Neuron $38: 433-445$.

814 24. Hutt A and Buhry L (2014) Study of GABAergic extra-synaptic tonic inhibition in single neurons

815 and neural populations by traversing neural scales: application to propofol-induced anaesthesia.J.

816 Comput. Neurosci. 37(3) : 417-437.

817 25. Amari S (1977) Dynamics of Pattern Formation in Lateral-Inhibition Type Neural Fields. Biol.

818 Cybern. $27: 77-87$

819 26. Lefebvre J, Hutt A, Knebel JF, Whittingstall K and Murray M (2015). Stimulus Statistics shape

820 oscillations in nonlinear recurrent neural networks. J. Neurosci. 35(7): 2895-2903

821 27. Herrmann C, Murray MM, Ionta S, Hutt A and Lefebvre J (2016) Shaping neural oscillations with 
822 periodic stimulation. J. Neurosci. 36(9): 5328-37

823 28. Wang XJ and Buzsaki G (1996). Gamma oscillation by synaptic inhibition in a hippocampal

824 interneuronal network model. J. Neurosci. 16(20) : 6402-6413

825 29. Fries P, Reynolds JH, Rorie AE, Desimone R (2001). Modulation of oscillatory neuronal

826 synchronization by selective visual attention. Science 291(5508):1560-3.

827 30. Lachaux JP, Rodriguez E, Martinerie J and Varela FJ (1999). Measuring phase synchrony in brain 828 signals. Human Brain Map. 8: 194-208

829 31. Hutt A and Munk MHJ (2007). Mutual phase synchronization in single trial data. Chaos and $830 \quad$ Complexity Letters 2 (2/3): 225-246.

831 32. Voytek B, Kayser AS, Badre D, Fegen D, Chang EF, Crone NE, Parvizi J, Knight RT and D’Esposito 832 M (2015). Oscillatory dynamics coordinating human frontal networks in support of goal 833 maintenance. Nature Neurosci. 18:1318-1324. doi: 10.1038/nn.4071

834 33. Deco G, Jirsa VK, Robinson PA, Breakspear M and Friston K. (2008). The dynamic brain: from 835 spiking neurons to neural masses and cortical fields. PLoS Comput Biol. 4(8):e1000092. doi: 836 10.1371/journal.pcbi.1000092

837 34. Scheib MC (2017) Brainstem influence on thalamocortical oscillations during anesthesia 838 emergence. Front. Syst. Neurosci. $11: 66$.

839 35. Purdon PL, Pierce ET, Mukamel EA, Prerau MJ, Walsh JL, Wong KFK, Salazar-Gomez AF, Harrell 840 PG, Sampson AL, Cimenser A, Ching S, Kopell NJ, Tavares-Stoeckel C, Habeeb K, Merhar R and 841 Brown EN (2012). Electroencephalogram signatures of loss and recovery of consciousness from 842 propofol. Proc. Natl. Acad. Sci. USA 110(12): E1142-E1151

843 36. Sherman SM (2001). Tonic and burst firing: dual modes of thalamocortical relay. Trends 
845 37. McCormick DA and Bal T (1997). Sleep and arousal : thalamocortical mechanisms. Annu. Rev. $846 \quad$ Neurosci. 20: 185-215.

847 38. Livingstone MS and Hubel DH (1981). Effects of sleep and arousal on the processing of visual 848 information in the cat. Nature 291:554-561.

849 39. Zecharia AY and Franks NP (2009). General anesthesia and ascending arousal pathways. 850 Anesthesiology 111(4):695-6. doi: 10.1097/aln.0b013e3181b061bc.

851 40. Chander D, Garcia PS, MacColl JN, Illing S and Sleigh JW (2014). Electroencephalographic 852 variation during end maintenance and emergence from surgical anesthesia. PLoS One. 853 9(9):e106291. doi: 10.1371/journal.pone.0106291.

854 41. Ghosh A, Rho Y, McIntosh AR, Kötter R and Jirsa VK (2008). Noise during Rest Enables the 855 Exploration of the Brain Dynamic Repertoire. PLoS Comput. Biol. 4(10): e1000196. Doi : 856 10.1371/journal.pcbi.1000196

857 42. Tononi G, Boly M, Massimini $M$ and Koch C (2014) Integrated information theory: from 858 consciousness to its physical substrate. Nature Rev. Neurosci. 17 (7): 450-461. 859 doi:10.1038/nrn.2016.44

860 43. Massimini M, Ferrarelli F, Huber R, Esser SK, Singh H and Tononi G (2005) Breakdown of 861 Cortical Effective Connectivity During Sleep. Science $309: 2228$.

862 44. Wollstadt P, Sellers KK, Rudelt L, Priesemann V, Hutt A, Frohlich F and Wibral MI 863 (2017). Breakdown of local information processing may underlie isoflurane anesthesia effects. 864 PLoS Comp. Biol. 13(6): e1005511. doi :10.1371/journal.pcbi.1005511

865 45. Hudetz AG, Liu X, Pillay S (2015) Dynamic repertoire of intrinsic brain states is reduced in 
propofol-induced unconsciousness. Brain Connect. 5(1):10-22. doi: 10.1089/brain.2014.0230

867 46. Stein RB, Gossen ER and Jones KE (2005). Neuronal variability : noise or part of the signal?

868 Nature Rev. Neurosci. 6 :389-397. doi : 10.1038/nrn1668

869 47. Fuller P, Sherman D, Pedersen NP, Saper CB and Lu J (2011) Reassessment of the structural 870 basis of the ascending arousal system. J. Comp. Neurol. 519(5): 933-956

871 48. Hudson AE, Calderon DP, Pfaff DW and Proekt A (2014). Recovery of consciousness is mediated 872 by a network of discrete metastable activity states. Proc. Natl. Acad. Sci. USA 111(25): 9283-9288.

873 doi: $10.1073 /$ pnas.1408296111

874 49. Schwappach C, Hutt A and beim Graben P (2015). Metastable dynamics in heterogeneous neural 875 fields. Front. Syst. Neurosci. 9:97. doi: 10.3389/fnsys.2015.00097

876 50. Vijayan S, Ching S, Purdon PL, Brown EN and Kopell NJ (2013). Thalamocortical Mechanisms for 877 the Anteriorization of Alpha Rhythms during Propofol-Induced Unconsciousness. J. Neurosci.

878 33(27):11070 -11075

879 51. Hashemi M, Hutt A and Sleigh J (2015). How the cortico-thalamic feedback affects the EEG 880 power spectrum over frontal and occipital regions during propofol-induced anaesthetic sedation. J.

881 Comput. Neurosci. 39(1): 155

882 52. Huang Z, Liu X, Mashour GA and Hudetz AG (2018) Timescales of Intrinsic BOLD Signal 883 Dynamics and Functional Connectivity in Pharmacologic and Neuropathologic States of 884 Unconsciousness. J. neurosci. 38(9) :2304-2317.

885 53. Li D, Voss LJ, Sleigh JW and Li X. (2013) Effects of volatile anesthetic agents on cerebral cortical 886 synchronization in sheep. Anesth. 119(1):81-8.

88754 . Liu X, Pillay S, Li R, Vizuete JA, Pechman KR, Schmainda KM and Hudetz AG (2013) Multiphasic 888 modification of intrinsic functional connectivity of the rat brain during increasing levels of propofol. 
890 55. Altweg-Boussac T, Schramm AE, Ballestero J, Grosselin F, Chavez M, Leca S, Baulac M, Naccache 891 N, Demeret S, Navarro V, Mahon S, Charpier S (2017). Cortical neurons and networks are dormant 892 but fully responsive during isoelectric brain state. Brain 140:2381-2398.

893 56. Fuller PM, Sherman D, Pedersen NP, Saper CB and Lu J (2011) Reassessment of the Structural 894 Basis of the Ascending Arousal System. J. Comp. Neurol. 519:933-956.

895 